\title{
Radiation Transfer Model Intercomparison (RAMI) exercise: Results from the second phase
}

\author{
B. Pinty, ${ }^{1}$ J.-L. Widlowski, ${ }^{1}$ M. Taberner, ${ }^{1}$ N. Gobron, ${ }^{1}$ M. M. Verstraete, ${ }^{1}$ M. Disney, ${ }^{2}$ \\ F. Gascon, ${ }^{3}$ J.-P. Gastellu, ${ }^{4}$ L. Jiang, ${ }^{5}$ A. Kuusk, ${ }^{6}$ P. Lewis, ${ }^{2}$ X. Li,${ }^{5}$ W. Ni-Meister, ${ }^{7}$ \\ T. Nilson, ${ }^{6}$ P. North, ${ }^{8}$ W. Qin,,${ }^{9}$ L. Su, ${ }^{5}$ S. Tang, ${ }^{5}$ R. Thompson, ${ }^{10}$ W. Verhoef, ${ }^{11}$ H. Wang, ${ }^{5}$ \\ J. Wang, ${ }^{5}$ G. Yan, ${ }^{5}$ and H. Zang ${ }^{5}$
}

Received 16 October 2003; revised 19 January 2004; accepted 4 February 2004; published 25 March 2004.

[1] The Radiation Transfer Model Intercomparison (RAMI) initiative is a communitydriven exercise to benchmark the models of radiation transfer (RT) used to represent the reflectance of terrestrial surfaces. Systematic model intercomparisons started in 1999 as a self-organized, open-access, voluntary activity of the RT modeling community. The results of the first phase were published by Pinty et al. [2001]. The present paper describes the benchmarking protocol and the results achieved during the second phase, which took place during 2002. This second phase included two major components: The first one included a rerun of all direct-mode tests proposed during the first phase, to accommodate the evaluation of models that have been upgraded since, and the participation of new models into the entire exercise. The second component was designed to probe the performance of three-dimensional models in complex heterogeneous environments, which closely mimic the observations of actual space instruments operating at various spatial resolutions over forest canopy systems. Phases 1 and 2 of RAMI both confirm not only that a majority of the radiation transfer models participating in RAMI are in good agreement between themselves for relatively simple radiation transfer problems but also that these models exhibit significant discrepancies when considering more complex but nevertheless realistic geophysical scenarios. Specific recommendations are provided to guide the future of this benchmarking program (Phase 3 and beyond). INDEX TERMS: 3399 Meteorology and Atmospheric Dynamics: General or miscellaneous; 3367 Meteorology and Atmospheric Dynamics: Theoretical modeling; 3360 Meteorology and Atmospheric Dynamics: Remote sensing; 3322 Meteorology and Atmospheric Dynamics: Land/atmosphere interactions; KEYWORDS: radiation transfer models, RAMI intercomparison exercise, surface radiation

Citation: Pinty, B., et al. (2004), Radiation Transfer Model Intercomparison (RAMI) exercise: Results from the second phase, J. Geophys. Res., 109, D06210, doi:10.1029/2003JD004252.

\section{Introduction}

[2] The proper interpretation of satellite remote sensing data (in particular but not exclusively over terrestrial surfaces) hinges on the availability of radiation transfer (RT) models that can be inverted against the measurements to retrieve the information of interest. The accuracy and reliability of the solutions to this inverse problem are determined by the performance of both the RT models

\footnotetext{
${ }^{1}$ Global Vegetation Monitoring Unit, Institute for Environment and Sustainability, EC Joint Research Centre, Ispra, Italy.

${ }^{2}$ Center for Terrestrial Carbon Dynamics, Department of Geography, University College London, London, UK.

${ }^{3}$ Land Surface Unit, European Space Agency, Noordwijk, Netherlands.

${ }^{4}$ Centre d'Études Spatiales de la Biosphère, Toulouse, France.

${ }^{5}$ Department of Geography, Beijing Normal University, Beijing, China.

Copyright 2004 by the American Geophysical Union.

0148-0227/04/2003JD004252\$09.00
}

and the remote sensing instruments. Indeed, the "goodness-of-fit" metrics used in the inversion procedure should incorporate, in principle, both an estimation of the reliability of the measurements and an assessment of the performance of the model.

[3] Recent advances in remote sensing technologies, in particular with respect to higher spectral and directional sampling capabilities, have stimulated the development of better models, allowing the retrieval of more detailed environmental information from these observations. The

\footnotetext{
${ }^{6}$ Tartu Observatory, Tõravere, Estonia.

${ }^{7}$ Department of Geography, Hunter College, City University of New York, New York, USA.

${ }^{8}$ Climate and Land Surface Systems Interaction Centre, Department of Geography, University of Wales, Swansea, UK.

${ }^{9}$ Science Systems and Applications, Inc., Greenbelt, Maryland, USA.

${ }^{10}$ Alachua Research Institute, Alachua, Florida, USA.

${ }^{11}$ Remote Sensing Department, National Aerospace Laboratory NLR, Emmeloord, Netherlands.
} 
RT modeling community is thus motivated to improve its offerings, and in particular to ensure that both the conceptual design and the numerical implementation of these models can take full advantage of these new advanced sensors. At the very least, model developers are interested in verifying that their codes perform adequately for a range of well-documented conditions. This is one of the prime motivations for RAMI, which is designed to measure the variability between existing models, to document their accuracy and reliability in simulating wellcontrolled experimental conditions and to provide diagnostics suitable to pinpoint possible deficiencies in these codes.

[4] The motivation for and methodology of the RAMI benchmarking exercise was inspired, in part, by a parallel activity in the cloud modeling community, known as the "Intercomparison of 3D Radiation Codes" (I3RC). As explained on the corresponding web site (http://I3RC. gsfc.nasa.gov/), this effort intends to understand and document the errors and limitations of 3-D models of radiation transfer in the atmosphere, to provide "baseline" cases to test 3-D codes and stimulate future developments, to promote the exchange of 3-D radiation tools, to propose guidelines for the selection of such models, and to improve atmospheric science education in this field.

[5] The basic principles of radiation transfer within the atmosphere and plant canopies are quite similar. Nevertheless, the latter is characterized by specific features that require particular care [e.g., Knyazikhin and Marshak, 1991; Pinty and Verstraete, 1998; Knyazikhin et al., 2004]. For instance, the extinction coefficient of light in plant canopies is independent of wavelength in the solar domain [Ross, 1981], the "far-field approximation" is not valid for terrestrial environments because of the finite size of the oriented scattering elements (soil grains and plant leaves [e.g., Irvine, 1966; Verstraete, 1987; Nilson and Kuusk, 1989; Verstraete et al., 1990; Knyazikhin et al., 1992]), the differential scattering coefficient is not rotationally invariant because of the preferred orientation of the scatterers [e.g., Shultis and Myneni, 1988], and the distribution of these scattering elements in three-dimensional space is not dictated by statistical thermodynamics and turbulence laws, so that three-dimensional models may be required to explicitly describe the position, size, shape, orientation and optical properties of each scattering element, at least for the more demanding applications.

[6] The RAMI benchmarking protocols were designed around a limited set of modeling exercises for both structurally homogeneous and heterogeneous terrestrial surfaces. This setup allows evaluating the implications of these surface-specific features. RAMI also proposes optional exercises to verify model compliance with respect to energy conservation under conservative conditions.

[7] The first phase of RAMI took place in the spring of 1999 and the results were unveiled at the "Second International Workshop on Multiangular Measurements and Models (IWMMM-2)" held at the Joint Research Centre (JRC, Ispra, Italy) on 15-17 September 1999. The overall objectives, rationale and strategy for the RAMI exercise, as well as a selection of benchmark results were published by Pinty et al. [2001] and are available at the following internet address: http://www.enamors.org/ RAMI/rami.htm.

[8] The second phase of RAMI was organized during the spring and summer of 2002 and results were first presented publicly at the "Third International Workshop on Multiangular Measurements and Models (IWMMM-3)" organized jointly by the National Snow and Ice Data Center and Jet Propulsion Laboratory in Steamboat Springs on June 10-12, 2002. RAMI Phase 2 was structured around two components: The first consisted in a repetition of all tests in direct mode that were part of Phase 1, offering the possibility to re-evaluate models that have been upgraded since, as well as to accommodate new models. The second component of Phase 2 was designed to test the performance of three-dimensional models for significantly heterogeneous environments. The latter mimic observations that could be provided by remote sensing instruments operating at various spatial resolutions over a forest canopy. The design of this series of experiments followed the conclusions and recommendations of the first phase and reflects the importance of coniferous tree forests for global and regional climate modeling.

[9] The next section describes the benchmarking protocol followed during RAMI Phase 2 and includes a detailed description of the heterogeneous scenes specifically designed for this phase. Subsequent sections analyze the results of the benchmarks and outline the major findings. The paper ends with a possible road map for RAMI and discusses future plans to evaluate the scientific advances of the radiation transfer modeling community.

\section{The RAMI Phase 2 Protocol}

[10] The main benchmarking protocol and underlying principles of the Phase 2 of RAMI followed those adopted earlier for Phase 1 (see section 2.1 of Pinty et al. [2001]). The coordination of the activity, the design of the experiments, the collection of the RT model results and their detailed analysis were performed at the Joint Research Center (Ispra, Italy). After an initial public presentation of the results of Phase 2 at the IWMMM-3 conference in June 2002, it was decided to extend the deadline for submitting simulation results for this second phase to October 2002, to accommodate trivial corrections and simple improvements that could be implemented immediately.

[11] Table 1 lists the models that participated in RAMI Phase 2, the main publications where these models are documented and the names and affiliations of the participants. It must be noted that the participating threedimensional models implement a wide range of approaches to simulate the radiation transfer regime in structurally heterogeneous scenes, including ray tracing techniques associated with inverse/direct Monte-Carlo (Flight, Sprint-2, Raytran, and Drat), and discrete ordinate methods (DART), radiosity (RGM), as well as hybrid, i.e., combining analytical and numerical solutions for random distributions of plant stands (GORT, SGORT, LIM and FRT). The participants received substantial feedback on their performance as a result of Phase 1 and to a limited extent in Phase 2, as explained above. Consequently, all results presented below refer to the latest and most up-to- 
Table 1. List of the RAMI Phase 2 Models, References, and Participants

\begin{tabular}{|c|c|c|}
\hline Model Name & References & Participants \\
\hline \multicolumn{3}{|c|}{ Structurally Homogeneous Scenes } \\
\hline SailH & Verhoef [1984, 1985] & W. Verhoef ${ }^{a}$ \\
\hline Sail++ & Verhoef $[1998,2002]$ & W. Verhoef ${ }^{\mathrm{a}}$ \\
\hline 1/2 Discrete & Gobron et al. [1997] & N. Gobron ${ }^{\mathrm{b}}$ \\
\hline \multicolumn{3}{|c|}{ Three-Dimensional Heterogeneous Scenes } \\
\hline Flight & North [1996] & P. North ${ }^{\mathrm{c}}$ \\
\hline DART & Gastellu-Etchegorry et al. [1996] & F. Gascon ${ }^{\mathrm{d}}$ and J.-P. Gastellu ${ }^{\mathrm{e}}$ \\
\hline Sprint-2 & Thompson and Goel [1998] & R. Thompson ${ }^{\mathrm{f}}$ \\
\hline Raytran & Govaerts and Verstraete [1998] & J.-L. Widlowski ${ }^{\text {b }}$ \\
\hline RGM & Qin and Gerstl [2000] & W. Qin ${ }^{\text {g }}$ \\
\hline Drat & Lewis [1999] and Saich et al. [2001] & M. Disney ${ }^{\mathrm{h}}$ and P. Lewis ${ }^{\mathrm{h}}$ \\
\hline GORT & Li et al. $[1995]$ & G. Yan, ${ }^{i}$ S. Tang, ${ }^{i}$ L. Jiang, ${ }^{i}$ H. Zang, ${ }^{i}$ H. Wang, ${ }^{i}$ J. Wang, ${ }^{i}$ and X. Li ${ }^{i}$ \\
\hline SGORT & Ni et al. [1999] & G. Yan, ${ }^{i}$ S. Tang, ${ }^{i}$ L. Jiang, ${ }^{i}$ H. Zang, ${ }^{i}$ H. Wang, ${ }^{i}$ J. Wang, ${ }^{i}$ and X. Li $^{i}$ \\
\hline LIM & unpublished & L. $\mathrm{Su}^{\mathrm{i}}$ \\
\hline FRT & Kuusk and Nilson [2000] & A. Kuusk ${ }^{\mathrm{j}}$ and T. Nilson ${ }^{\mathrm{j}}$ \\
\hline
\end{tabular}

${ }^{\mathrm{a}}$ National Aerospace Laboratory NLR.

${ }^{\mathrm{b} J o i n t ~ R e s e a r c h ~ C e n t r e . ~}$

${ }^{\mathrm{c}}$ Department of Geography, University of Wales, Swansea.

${ }^{\mathrm{d}}$ Land Surface Unit, European Space Agency.

${ }^{\text {e}}$ Centre d'Études Spatiales de la Biosphère.

${ }^{\mathrm{f}}$ Alachua Research Institute.

gNASA Goddard Space Flight Center.

${ }^{h}$ Department of Geography, University College London.

${ }^{\mathrm{i}}$ Department of Geography, Beijing Normal University.

${ }^{\mathrm{j}}$ Tartu Observatory, Tõravere.

date version of these models. It is important that prospective users of these models ensure that they have access to the most current version of these codes, as the performance information provided here may not be representative of or applicable to earlier versions.

[12] Phase 2 of RAMI expands on the benchmarking protocols of Phase 1. It proposes new experiments specifically designed to compare the performance of 3-D RT models in simulating (1) a forest of coniferous trees randomly scattered over a Gaussian-shaped surface and (2) a canopy composed of spectrally and structurally heterogeneous trees randomly located over a flat terrain, at various spatial resolutions.

[13] These two new experiments complement the earlier tests of Phase 1, which featured a simpler idealized threedimensional plant canopy composed of floating spheres filled with leaves (see section 2.1.2 of Pinty et al. [2001]). Exhaustive documentation on the spectral and structural properties of these plant canopies (including the exact location in three-dimensional (3-D) space of the clumps of scatterers) were delivered for all 3-D scenes to the participants. The latter were free to choose the level of simplification required to best represent these scenes within their models.

[14] For all proposed experiments, the participants were encouraged to generate, in both the principal and the cross planes, the total spectral bidirectional reflectance factor (BRF) values, the corresponding contributions due to the single uncollided radiation scattered once by the soil only, the single collided radiation by the leaves or trees only, and the radiation multiply collided by the leaves/trees-soil system. Additional quantities including the spectral albedo of the canopy, i.e., the directional hemispherical reflectance, and the absorption of radiation in the vegetation layer were also asked for. This ensemble of information proved very useful in assessing the strengths and weaknesses of the various modeling techniques used.

\subsection{Coniferous Forest Scene}

[15] This set of experiments was suggested specifically to test the ability of radiation transfer models to simulate spatially heterogeneous scenes containing some degree of topography in the red and near-infrared spectral bands.

[16] The canopy architecture and spectral properties were chosen to reflect those of typical coniferous forests. A large number of nonoverlapping, partially covering, tree-like entities (composed of a conically shaped crown located above a cylindrical trunk) were randomly distributed across a Gaussian-shaped surface that represented the underlying soil topography. The total scene size covered a square area $500 \mathrm{~m}$ on the side. Within this scene, the $(x, y)$ location of these tree-like entities was determined by a Poisson distribution. For each given $(x, y)$, the elevation of the hill, which is also the base of the trunk $(z)$, was then computed using an elevation formula. This formula provided a Gaussianshaped height field with a maximum elevation value of $100 \mathrm{~m}$ at the scene center $(0,0)$ and elevation values very close to zero at the edges of the scene.

[17] The foliage itself, composed of a finite number of scattering centers implemented as small disks, was randomly distributed within the conical volumes that represented the tree crowns, and was characterized by specific radiative properties (reflectance, transmittance) as well as the orientation distribution function for the normals to the scatterers (uniform distribution). The bright underlying surface was meant to render a snow-covered ground typical of winter conditions, albeit represented by a simple Lambertian law.

[18] Tables 2 and 3 provide the values for the relevant architectural and spectral variables, respectively, to be used 
Table 2. Variables Defining the Structure of the Coniferous Tree Scene

\begin{tabular}{ll}
\hline \multicolumn{1}{c}{ Variable Identification } & \multicolumn{1}{c}{ Values } \\
\hline Scatterer shape & disc of negligible thickness \\
Scatterer radius & $0.05 \mathrm{~m}$ \\
Leaf area index of a tree crown & $5 \mathrm{~m}^{2} / \mathrm{m}^{2}$ \\
Scatterer normal distribution in tree crown & uniform \\
Total number of trees in scene & $10^{4}$ \\
Spatial distribution of tree locations & Poisson distribution \\
Tree density & 400 trees $/$ hectare \\
Fractional scene coverage of cones & 0.407 \\
Leaf area index of the scene & $2.036 \mathrm{~m}^{2} / \mathrm{m}^{2}$ \\
Tree crown length & $12.00 \mathrm{~m}$ \\
Tree crown-base width & $3.60 \mathrm{~m}$ \\
Tree trunk height & $1.50 \mathrm{~m}$ \\
Tree trunk diameter & $0.30 \mathrm{~m}$ \\
Minimum elevation of the terrain & $0.00 \mathrm{~m}$ \\
Maximum elevation of the terrain & $100.00 \mathrm{~m}$ \\
\hline
\end{tabular}

in the proposed simulations. The illumination zenith angle was fixed at $40^{\circ}$.

[19] Figures $1 \mathrm{a}$ and $1 \mathrm{~b}$ exhibit graphical representations of this scene when viewed from the side and from the top, respectively.

\subsection{Structurally and Spectrally Mixed Vegetation Scene}

[20] A second set of new experiments was suggested to exercise the models in simulating the radiative transfer regime within a highly heterogeneous canopy at various spatial resolutions in the near-infrared spectral band. The idealized tree stand was composed of spherical or cylindrical volumes containing large numbers of disc-shaped scatterers. These individual volumes, meant to represent individual tree crowns, were nonoverlapping and distributed over an otherwise flat and relatively dark horizontal surface.

[21] The scatterers themselves were randomly distributed within these volumes and characterized by appropriate spectral (reflectance and transmittance) and geometric (position and orientation, in this case uniform) variables. The underlying soil was again simulated as a simple Lambertian reflector and the illumination zenith angle was fixed at $20^{\circ}$. Tables 4 and 5 provide the necessary information to perform this experiment.

[22] The reflectance of this heterogeneous discrete scene had to be computed at three different spatial resolutions; that is, all RAMI computations had to be derived for three progressively smaller target areas, each located at the center of the previous one. The larger target covered an area of $270 \times$ $270 \mathrm{~m}^{2}$, while the other two concerned $90 \times 90 \mathrm{~m}^{2}$ and $30 \times$ $30 \mathrm{~m}^{2}$, respectively. It was also suggested to consider the illumination of the entire $270 \times 270 \mathrm{~m}^{2}$ scene even when the RAMI measurements were made for the smaller target areas.

Table 3. Variables Defining the Spectral Leaf and Soil Properties of the Coniferous Tree Scene

\begin{tabular}{lcc}
\hline \multicolumn{1}{c}{ Variable Identification } & Red Values & $\begin{array}{c}\text { Near-Infrared } \\
\text { Values }\end{array}$ \\
\hline Leaf scatterer reflectance within cones $^{\mathrm{a}}$ & 0.080 & 0.450 \\
Leaf scatterer transmittance within cones $^{\mathrm{a}}$ & 0.030 & 0.300 \\
Trunk reflectance $^{\mathrm{b}}$ & 0.140 & 0.240 \\
Soil reflectance $^{\mathrm{b}}$ & 0.860 & 0.640
\end{tabular}

${ }^{\mathrm{a}}$ Using a bi-Lambertian scattering law.

${ }^{\mathrm{b}}$ Using a Lambertian scattering law.
[23] Figures $2 \mathrm{a}$ and $2 \mathrm{~b}$ exhibit graphical representations of this scene when viewed from the side and from the top, respectively. The red squares on the three subpanels in Figure $2 \mathrm{~b}$ visually indicate the areal extent of the three spatial resolutions.

\section{Overview of RAMI Phase 2 Results}

[24] The participating groups performed a large number of radiation transfer computations. These were carefully analyzed and a rather exhaustive set of results is publicly available from the following internet site: http://www. enamors.org/RAMI/rami.htm. In this paper, only the most prominent results obtained during the second phase of RAMI will be highlighted.

[25] The first subsection reviews the results obtained by the participants in Phase 2 while carrying the experiments involving finite-size scatterers proposed in the first phase of RAMI. In this case, the expected results were already known, since they had been published in 2000 , but this

\section{Coniferous tree forest}

$$
\text { (a) - Side view }
$$

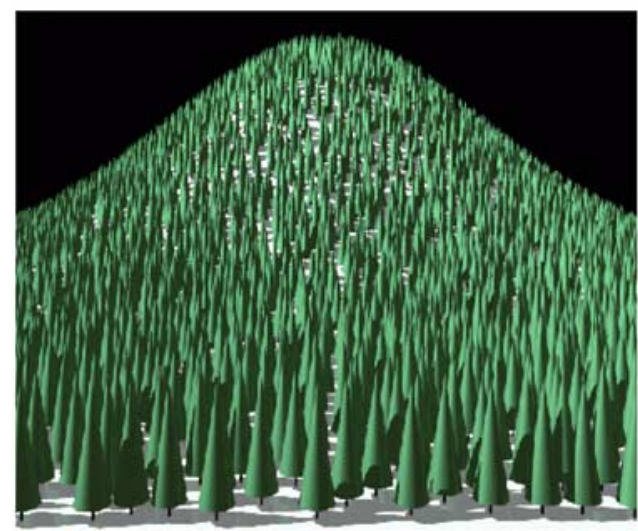

(b) - Top view

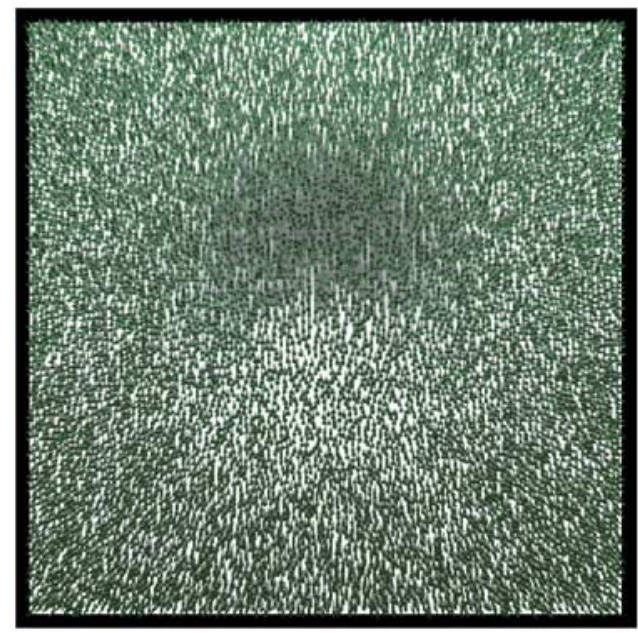

Figure 1. Artist representations of the RAMI Phase 2 coniferous trees scenes when (a) looking from across its "eastern" edge in a "westward" direction toward the center of the scene and (b) looking vertically down toward the center of the scene. The Sun is assumed located "south" of the scene. 
Table 4. Major Variables Defining the Structure of the Mixed Vegetation Scene

\begin{tabular}{ll}
\hline \multicolumn{1}{c}{ Variable Identification } & \multicolumn{1}{c}{ Values } \\
\hline Leaf scatterer shape & disc of negligible thickness \\
Leaf scatterer radius & $0.05 \mathrm{~m}$ \\
Leaf area index of individual spheres & $5 \mathrm{~m}^{2} / \mathrm{m}^{2}$ \\
Leaf area index of individual cylinders & $5 \mathrm{~m}^{2} / \mathrm{m}^{2}$ \\
Leaf scatterer normal distribution & uniform \\
Number of spheres & 205 \\
Number of cylinders & 409 \\
Fractional scene coverage of spheres & 0.141 \\
Fractional scene coverage of cylinders & 0.159 \\
Combined fractional scene area coverage & 0.30 \\
Sphere radius & $4.00 \mathrm{~m}$ \\
Cylinder radius & $3.00 \mathrm{~m}$ \\
Cylinder height & $12.00 \mathrm{~m}$ \\
Maximum height of top of the canopy & $15.00 \mathrm{~m}$ \\
\hline
\end{tabular}

provided an opportunity to update the statistics as models participating in Phase 1 had been upgraded and new models recently joined the exercise.

[26] The second subsection below summarizes the results derived from the new experiments described above, namely the modeling of the radiation transfer regime in threedimensional structurally heterogeneous environments over hilly terrain and at various spatial resolutions. The RAMI steering group at JRC maintained contact with all participants and provided limited feedback when doubtful results probably indicated obvious errors in model implementation or in scene representation. Other than that, all simulations were made blindly, in the sense that the individual participants did not know a priori who else was performing the RAMI Phase 2 exercises, which models were used, which experiments were made, or what results to expect. Five RT models, namely Sprint-2, Raytran, Drat, DART and FRT, participated in the new 3-D specific component of the second phase of RAMI. As mentioned previously, these models are quite different from each other in design and implementation. They span a wide range of approaches and techniques currently popular to simulate the transfer of radiation in structurally heterogeneous plant canopy systems.

\subsection{RAMI Phase 1 Structurally Homogeneous Scenes}

[27] The two sets of experiments proposed to exercise RT models over structurally homogeneous scenes constitute baseline cases in the context of the RAMI initiative. They allow us to benchmark the performance of models implementing a plane-parallel one-dimensional solution, both between themselves and in comparison with the models that solve the RT equation explicitly in the three spatial dimensions. These structurally homogeneous scenes are made up of finite-size scatterers with anisotropic scattering

Table 5. Variables Defining the Spectral Leaf and Soil Properties of the Mixed Vegetation Scene

\begin{tabular}{lc}
\hline \multicolumn{1}{c}{ Variable Identification } & Near-Infrared Values \\
\hline Leaf scatterer reflectance within spheres $^{\mathrm{a}}$ & 0.490 \\
Leaf scatterer transmittance within spheres $^{\mathrm{a}}$ & 0.410 \\
Leaf scatterer reflectance within cylinders $^{\mathrm{a}}$ & 0.450 \\
Leaf scatterer transmittance within cylinders $^{\mathrm{a}}$ & 0.300 \\
Soil reflectance $^{\mathrm{b}}$ & 0.150 \\
\hline
\end{tabular}

${ }^{a}$ Using a bi-Lambertian scattering law.

${ }^{\mathrm{b}}$ Using a Lambertian scattering law. properties randomly distributed between two horizontal planes representing the top of the canopy (the average level relevant to define the incoming downward radiation from the source and the upward exiting radiation scattered by the plant canopy system) and the bottom of the canopy (the level where radiation reaches the lower boundary condition, i.e., the soil background). The number and characteristics of the scatterers located between these two planes, as well as the properties of the lower boundary conditions, are varied to exercise RT models for different leaf area index and soil background conditions (in two spectral bands).

[28] One of these two sets, identified as the "purist corner" experiment, regarded conservative scattering and was proposed to verify the compliance of the participating models with respect to energy conservation. This exercise proved indeed very useful not only for verifying the behavior of the RT vegetation models under extreme conditions, but also for pointing out their deficiencies. In these cases, the scatterer reflectance and transmittance values are both equal to 0.5 and the soil reflectance is equal to 1.0 . The other variables included the leaf diameter, set at $0.1 \mathrm{~m}$, the leaf area index (fixed at $1.0,3.0$ and $5.0 \mathrm{~m}^{2} \mathrm{~m}^{-2}$ ) and the height of the plant canopy $(1 \mathrm{~m})$. Two leaf angle distribution functions, known as erectophile and planophile, are suggested for performing the simulations at three different illumination zenith angles $\left(0,30\right.$ and $\left.60^{\circ}\right)$. A total of $2736 \mathrm{BRF}$ values were produced by each participating model.

\section{Spectrally and structurally mixed Vegetation}

$$
\text { (a) - Side view }
$$

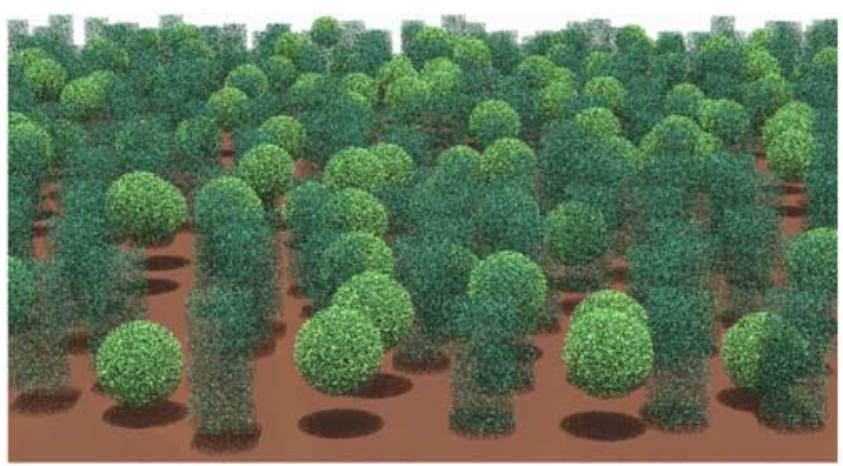

(b) - Top view
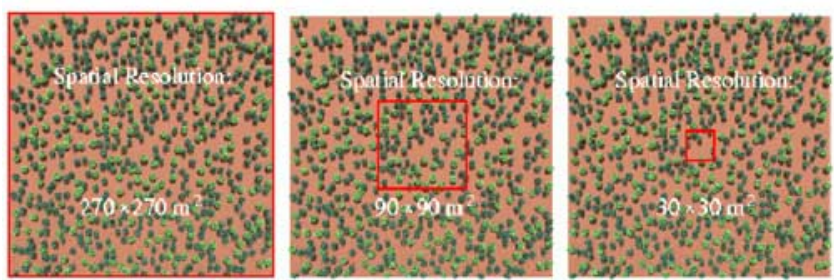

Figure 2. Artist representations of the RAMI Phase 2 mixed vegetation scenes when (a) looking from across "northern" edge in a "southward" direction toward the center of the scene and (b) looking vertically down toward the center of the scene. The red squares shown on the three bottom panels feature the three spatial resolutions considered successively in this set of experiments. The Sun is assumed located "north" of the scene. 
[29] The other set, identified simply as the "homogeneous vegetation scenes" experiments, covers the classical range of spectral conditions exhibited by live green vegetation at the red and near-infrared wavelengths. The exact conditions of the experiments to be performed as well as the full description of the scenes yielding the simulation of 912 BRF values was provided in section 2.1.1 of Pinty et al. [2001].

[30] An analysis of the results of this second phase of RAMI for the structurally homogeneous scenes was thus established on the basis of 3648 values of BRF delivered by each participating model. An ensemble of three 3-D and three 1-D models performed the entire sets of experiments proposed in the context of the homogeneous scenes, and further analyses were conducted to quantify intermodel variability. The behavior of each individual model with respect to the ensemble of RAMI models was examined using metrics identical to those adopted during RAMI Phase 1 (see equations (1), (2), and (3) in section 2.2 of Pinty et al. [2001]). One such metrics, called the local model deviation, is computed as follows:

$\delta_{m}\left(\theta_{v}\right)=\frac{200}{N_{l}} \sum_{i=1}^{N_{\theta_{0}}} \sum_{s=1}^{N_{\text {scenes }}} \sum_{\lambda=1}^{N_{\lambda}} \sum_{k=1, k \neq m}^{N_{\text {models }}} \frac{\left|\rho_{m}\left(\theta_{v}, i, s, \lambda\right)-\rho_{k}\left(\theta_{v}, i, s, \lambda\right)\right|}{\left[\rho_{m}\left(\theta_{v}, i, s, \lambda\right)+\rho_{k}\left(\theta_{v}, i, s, \lambda\right)\right]}$,

where $\delta_{m}\left(\theta_{v}\right)$ expresses, in percent, the local angular deviation or distance of model $m$ at the specific exiting angle $\theta_{v}$ with respect to the ensemble of $N_{\text {models }}$ models. This deviation, normalized by the number of cases considered $\left(N_{l}\right)$, is estimated for all simulations of the BRF fields, emerging from $N_{\text {scenes }}$ at $N_{\lambda}$ wavelengths, illuminated with $N_{\theta_{0}}$ incident source angles. The $\rho_{m}\left(\theta_{v}, i\right.$, $s, \lambda)$ and $\rho_{k}\left(\theta_{v}, i, s, \lambda\right)$ correspond to the BRF values generated by model $m$ and any of the other $k$ RAMI model participating in the experiment, respectively. The envelope of the histograms of the local deviation metrics value permits to assess the various modes of the distribution of the model discrepancies. Furthermore, the joint analysis of the individual histograms of the local deviations for every model also permits to quantify its behaviour against the others.

[31] Figure 3 shows, as a function of the viewing angle, the values of $\delta_{m}$ derived from equation (1). The $\delta_{m}\left(\theta_{v}\right)$ values were assessed on the basis of the $3648 \mathrm{BRF}$ values covering thus the full range of conditions suggested for the structurally homogeneous scenes. The bottom panel exhibits the $\delta_{m}\left(\theta_{v}\right)$ values obtained for the 3-D models that have performed the entire set of experiments proposed in the context of the structurally homogeneous scenes, namely, Flight, Sprint-2 and Raytran. Despite the use of quite stringent conditions for evaluating the relative performances of three types of different RT models, the level of agreement between these models is rather high. The largest deviations appear to increase when observing closer to nadir conditions but this feature may be artificially enhanced by the requirement to quantify the relative and not the absolute BRF deviations (in this latter case the end results would thus be essentially controlled by results from experiments realized with high-scattering conditions). These findings significantly increase our confidence in this subset of three-dimensional
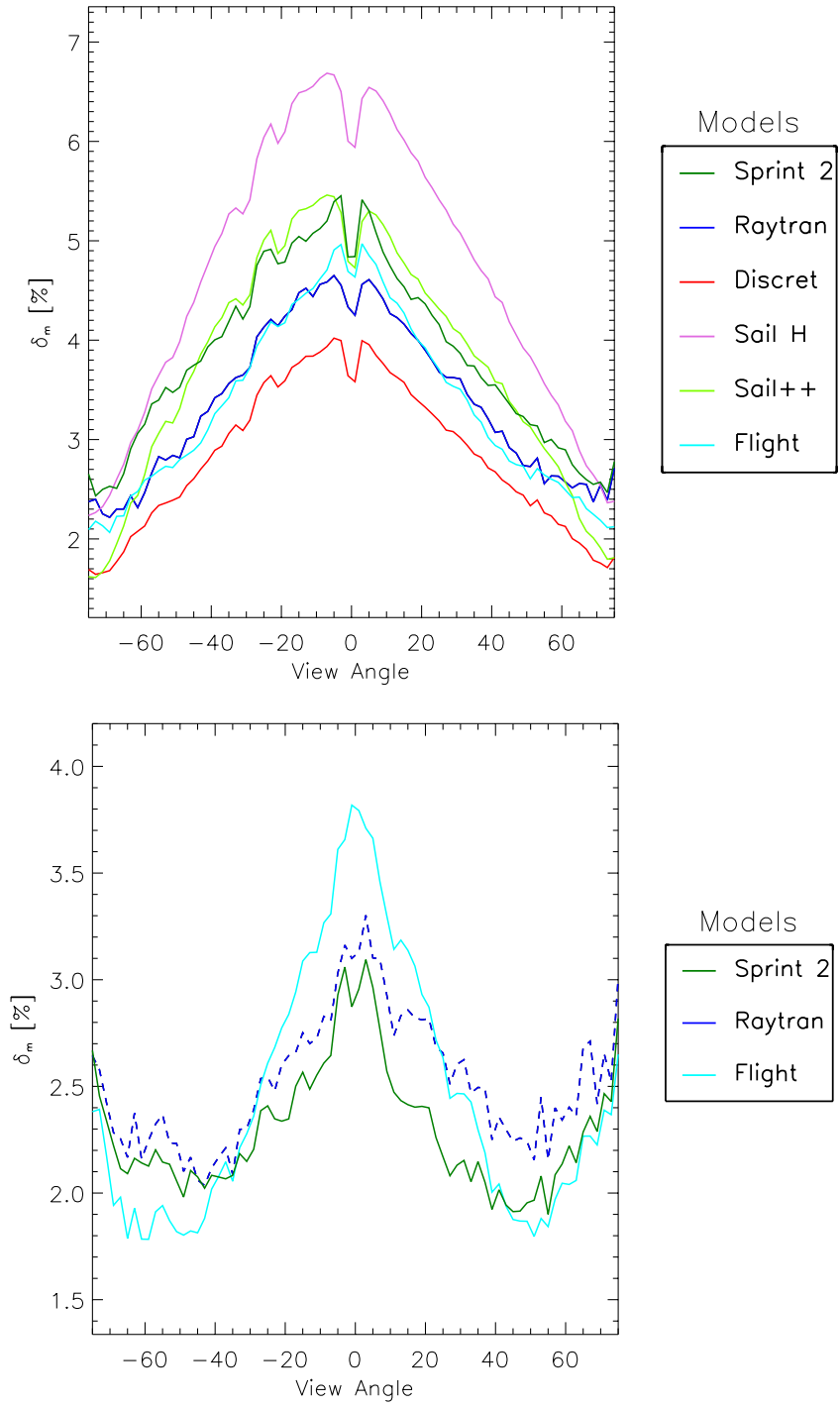

Figure 3. Variations of the local angular model deviation with respect to the 3648 total BRF values estimated from six 1-D and 3-D models (top panel) and three 3-D models (bottom panel) on the basis of equation (1); that is, all BRF values are accumulated over all illumination and viewing geometries, at two wavelengths and for all experimental conditions proposed for structurally homogeneous vegetation scenes that were also part of RAMI Phase 1.

RT models. The latter will thus serve to establish the most credible solutions (as a surrogate for the unknown truth) against which other RT models having performed a more limited set of experiments can be evaluated.

[32] Relative model performances can thus be assessed for a variety of scattering, illumination and observation conditions. Figures 4 and 5 show two examples of model behavior in the principal plane for the "homogeneous vegetation" and the "purist corner" scenes, respectively. These types of plots were instrumental to examine in detail the behavior of each individual model with respect to the ensemble of participating models. For instance, it turns out that the BRFs generated by Drat show a tendency to stay on the lower bound of the sets for the erectophile conditions. 
RED
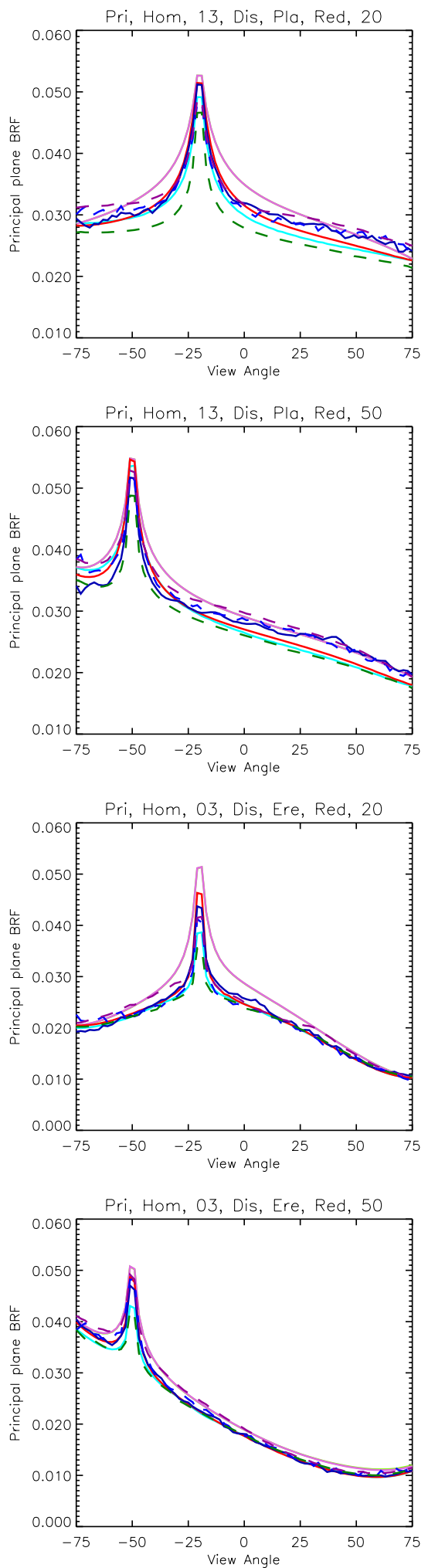

NIR
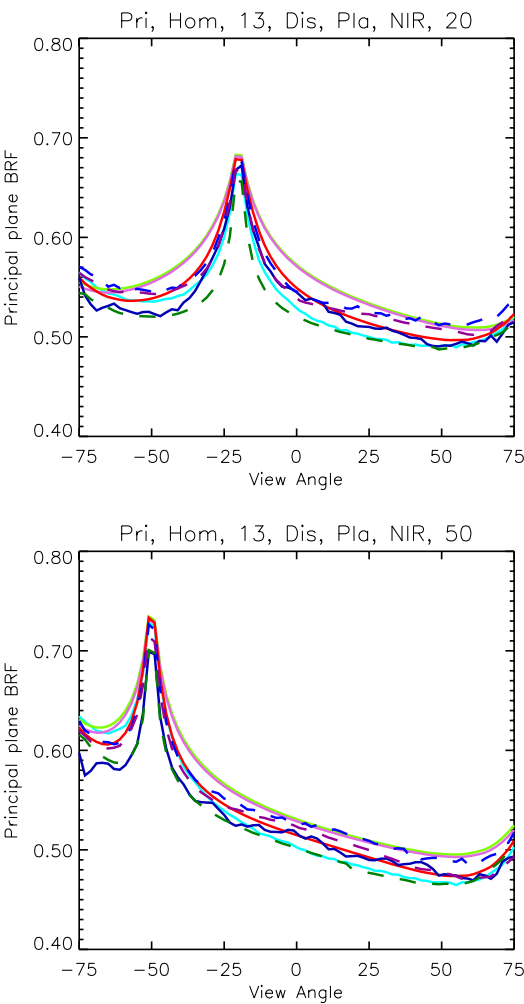

Models
\begin{tabular}{|ll|}
\hline - & Sprint 2 \\
- & RGM \\
- & Raytran \\
- & drat \\
- & Discret \\
- Sail H \\
- Sail++ \\
— & Flight \\
\hline
\end{tabular}

寔

Figure 4. BRF results from the RAMI models plotted in the principal plane for the red (left panels) and near-infrared (right panels) wavelengths. The top (bottom) four panels correspond to a homogeneous vegetation scene specified using a planophile (erectophile) leaf angle distribution function. The conditions of the experiments are given on top of each panel using the following convention: Dis stands for discrete medium, Pla (Ere) corresponds to planophile (erectophile) leaf angle distribution, and 20 (50) indicates the illumination zenith angle. 

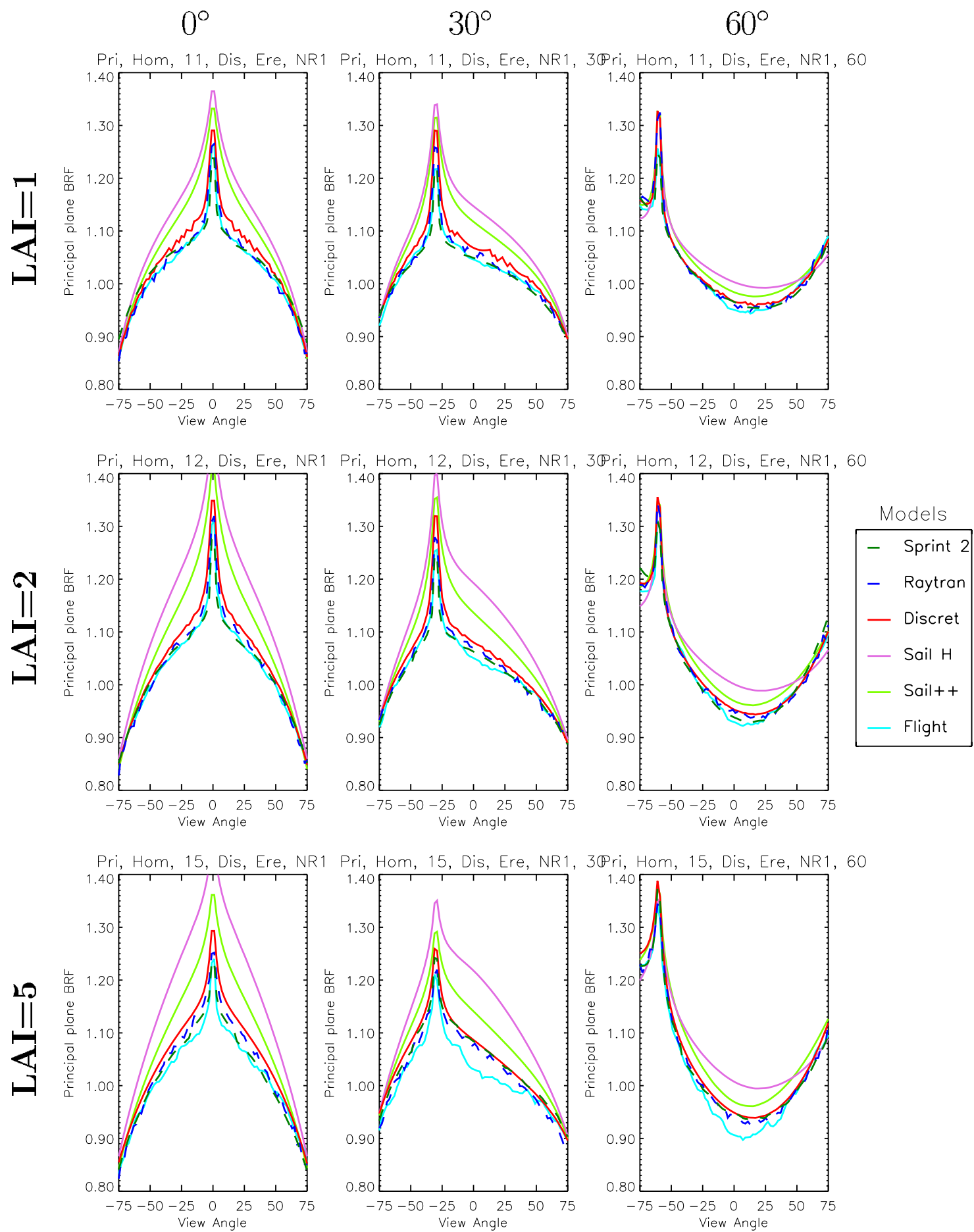

Figure 5. Plots of the BRFs in the principal plane for a homogeneous scene from the "purist corner," i.e., conservative scattering conditions, with an erectophile leaf angle distribution function. The top, middle, and bottom panels correspond to leaf area index values of 1,2 , and 5, respectively. The illumination zenith angle is set at values of $0^{\circ}$ (left panels), $30^{\circ}$ (middle panels), and $60^{\circ}$ (right panels). The conditions of the experiments are given on top of each panel using the same convention as before.

An analysis of the separate contributions to the total BRF values (not shown here) indicate that Flight generates multiple-scattering contributions that appear to cluster near the upper bound, specially at close to nadir exiting angles. The multiple-scattering contributions delivered by SailH in the case of erectophile leaf angle distribution are on the lower bounds while the uncollided contributions from both SailH and Sail++ are overestimated with respect to those produced by other models. This overestimation is related to the wider hot spot simulated by these models in comparison to those of the other models. In both SAIL models the hot spot is estimated from physically based, yet partly 


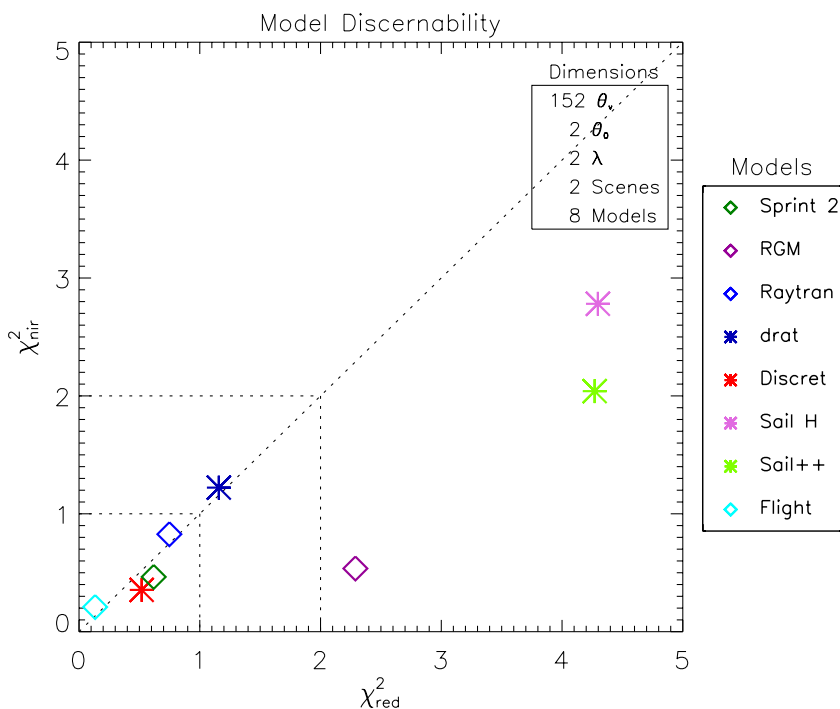

Figure 6. Plots of the $\chi^{2}(\lambda)$ values estimated using equation (2) for each of the RAMI Phase 2 models in the case of the structurally homogeneous vegetation scenes. All models falling within the subspace defined by $0<\chi^{2}(\lambda)<$ 1.0 are indistinguishable, on the basis of the available sample of measurements, within the one $\sigma(\lambda)$ range value.

parametric, considerations [Verhoef, 1998], and the width of the resulting hot spot is strongly related to the ratio of correlation length to canopy height. Normally, this correlation length is tentatively set equal to the leaf width, but it can be argued that in the case of circular leaves, half the diameter would have been a better approximation, and this would have resulted in a much narrower hot spot. The contribution of Sprint-2 in the single collided contribution is generally near the lower bound of the sets in the specific case of the planophile leaf angle distribution. The uncollided contributions computed from Raytran also appear located on the lower bound of the sets. In the case of Monte-Carlo ray-tracing models, however, the various ways adopted to distribute the collided and uncollided contributions may explain some of the intermodel differences for these specific components. This, however, does not affect the estimations of the total BRF values.

[33] The concept of model "discernability," defined as the ability to discern a model from another, was introduced in the first phase of RAMI (see section 3.3 of Pinty et al. [2001]). This is a key concept in this intercomparison context, as it basically permits to address practical issues such as the significance of differences obtained in inverse mode with an ensemble of RT models. In a pragmatic sense, differences between RT models matter only to the extent that they exceed the level of uncertainty associated with the measured BRF fields. It was thus proposed, during RAMI Phase 1, to adopt the "most credible solution," derived from the arithmetic mean of every BRF value calculated within a subset of the three-dimensional models, as a surrogate for the "truth." Detailed 3-D RT models, for instance based on Monte-Carlo ray tracing or similar techniques, represent the transfer of radiation in complex geophysical environments much more accurately than 1-D plane parallel models because they do not rely on simplifying assumptions, such as a parametric or statistical repre- sentations of the structure of plant canopies, empirical descriptions of the hot spot, etc. In this sense, the 3-D models that explicitly represent light-scatterer interaction processes constitute a useful standard against which to evaluate the parameterizations implemented in 1-D models. The subset of three-dimensional models must thus include, logically, models having performed all series of experiments related to the structurally homogeneous scenes. As can be seen from Figure 3, three RT models, namely Flight, Raytran and Sprint-2 are eligible for establishing the values of the "most credible solutions," as well as the uncertainties associated to these mean values. The model discernability with respect to the structurally homogeneous vegetation scenes can then be evaluated by comparing the values computed with the following normalized $\chi^{2}(\lambda)$ metrics:

$$
\chi^{2}(\lambda) \frac{1}{N-1} \sum_{i=1}^{N_{\theta_{0}}} \sum_{j=1}^{N_{\theta_{v}}} \sum_{s=1}^{N_{\text {scenes }}} \frac{\left[\rho(i, j, s, \lambda)-\rho^{\text {Credible }}(i, j, s, \lambda)\right]^{2}}{\sigma^{2}(\lambda)},
$$

with

$$
\rho^{\text {Credible }}(i, j, s, \lambda)=\left\langle\rho^{3 D}(i, j, s, \lambda)\right\rangle
$$

and

$$
\begin{aligned}
\sigma_{3 D}^{2}(\lambda)= & \frac{1}{N_{B}-1} \sum_{m=1}^{N_{3 D}} \sum_{i=1}^{N_{\theta_{0}}} \sum_{j=1}^{N_{\theta_{v}}} \sum_{s=1}^{N_{\text {scenes }}}\left[\rho^{3 D}(i, j, s, \lambda)\right. \\
& \left.-\rho^{\text {Credible }}(i, j, s, \lambda)\right]^{2} .
\end{aligned}
$$

Equations (3) and (4) provide an estimate of the average of the $N_{B}$ BRF values taken over a subset of $N_{3 D}=3$ threedimensional RAMI models and the associated value of the variance of the BRF distribution of these models. $N$ is a norm equal to the number of available cases. On the basis of the BRF values generated by the Flight, Raytran and Sprint-2 models, we obtained $\sigma_{3 D}(\lambda)$ values of 9.9E-04 and 9.0E-03 at the red and near-infrared wavelengths, respectively. These values correspond approximately to $2 \%$ of the typical BRF values that can be measured over a plant canopy system at these wavelengths. As was proposed during RAMI Phase 1, the $\sigma^{2}(\lambda)$ introduced at the denominator of equation (2), can be approximated by twice the values corresponding to the model uncertainty (considering that the uncertainties linked to the models are identical to those associated with the measurements):

$$
\sigma^{2}(\lambda)=2 \sigma_{3 D}^{2}(\lambda)
$$

The results of the application of the $\chi^{2}(\lambda)$ metrics defined by equation (2) are graphically shown in Figure 6. All models falling within the subspace defined by $0<\chi^{2}(\lambda)<1.0$ are statistically indistinguishable, on the basis of the available sample of measurements and within the prescribed level of uncertainty, e.g., one $\sigma(\lambda)$ range value corresponding to about $4 \%$ of the BRF values. The axes indicate the number of $\sigma(\lambda)$ values that has to be adopted for reaching the "indiscernability" limit. Figure 6 also illustrates the spectral biases of the RT model simulations; for instance, the RGM model delivers results that cannot be discerned from those falling within the subspace $0<\chi^{2}(\lambda)<1.0$ at the near- 
RED
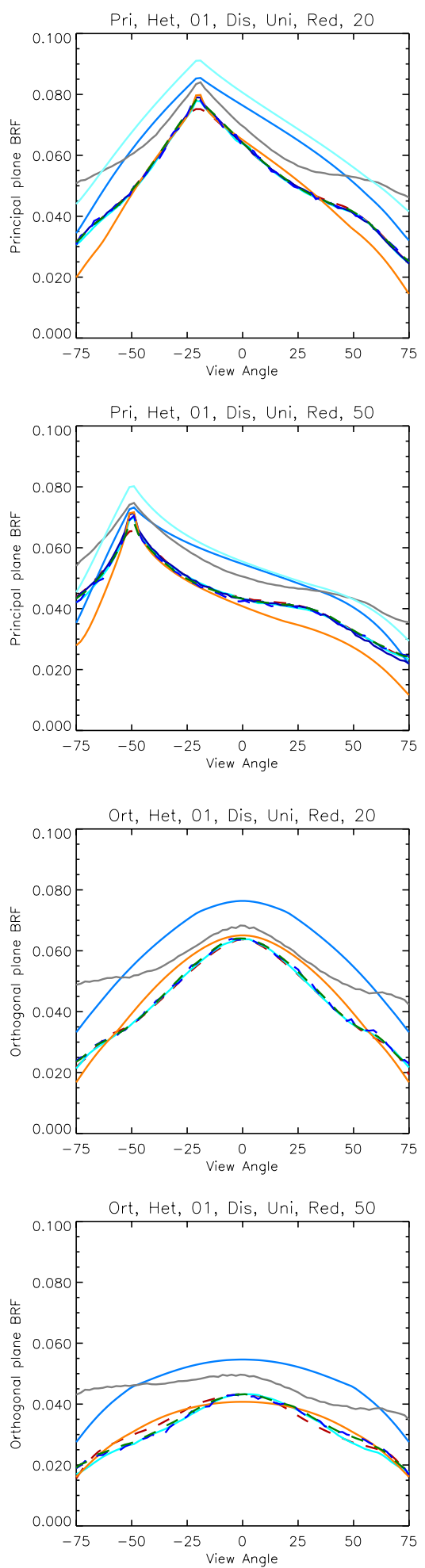

NIR
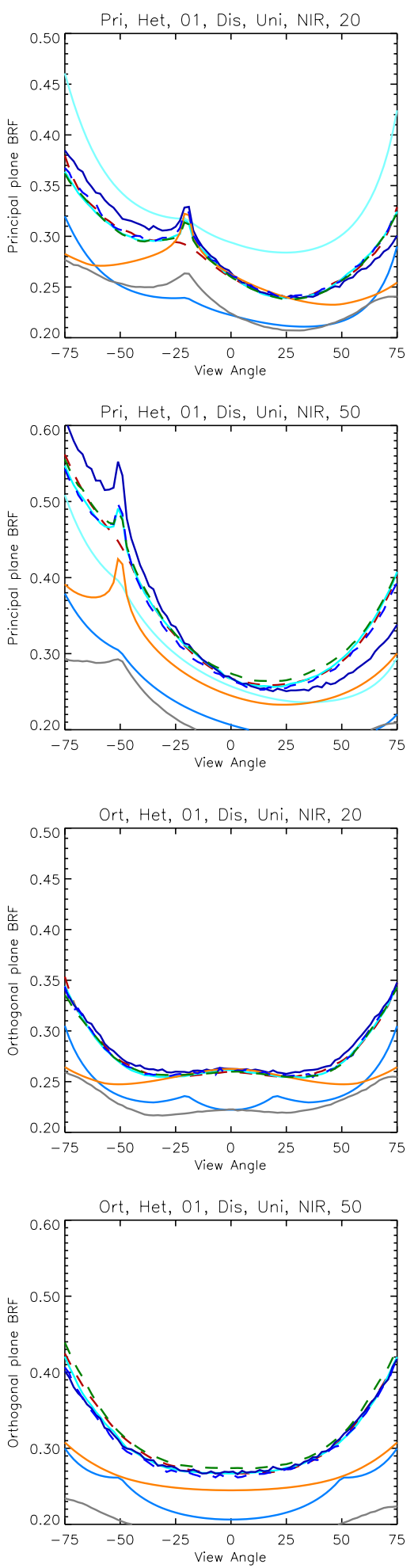

$\frac{0}{8}$

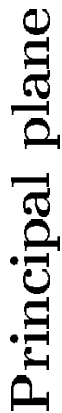

\begin{tabular}{|ll}
\multicolumn{1}{c}{ Models } \\
\hline- & Sprint 2 \\
- & Raytran \\
- & FRT \\
- & drat \\
- & SGORT \\
- & LIM \\
- & GORT \\
- & Flight \\
- & DART
\end{tabular}

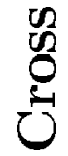

Figure 7. Total BRF results from the RAMI models plotted for the red (left panels) and near-infrared (right panels) wavelengths. The top (bottom) four panels show results generated in the principal (cross) plane at illumination zenith angles of $20^{\circ}$ and $50^{\circ}$, respectively. The conditions of the experiments are given on top of each panel using the following convention: Pri (Ort) identifies the principal (cross) plane, Het is for heterogeneous scene, Dis stands for discrete scatterers, Uni corresponds to uniform leaf angle distribution, and 20 (50) indicates the illumination zenith angle. Results correspond to simulations performed with respect to the floating sphere scene that was part of RAMI Phase 1. 

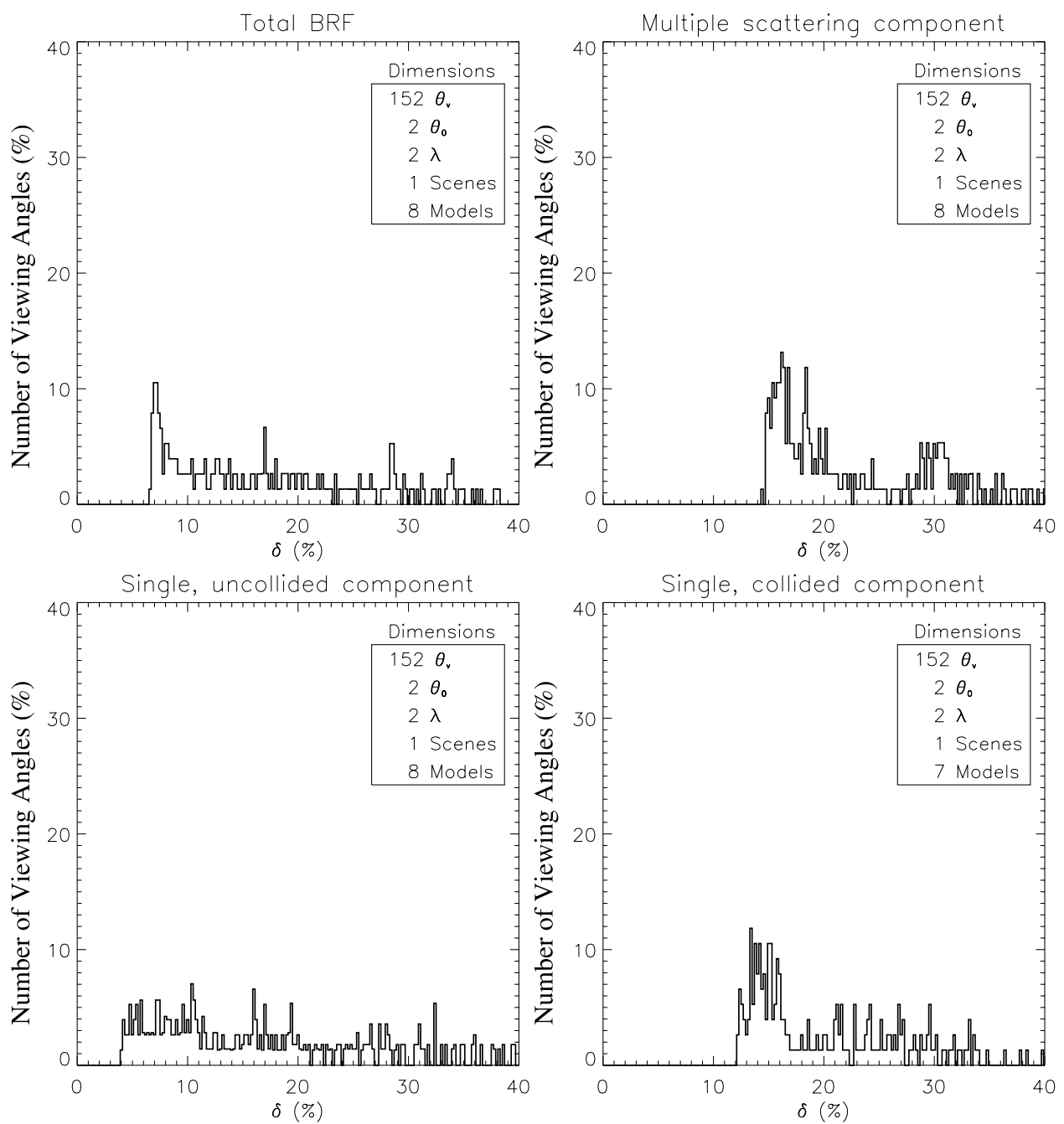

Figure 8. Histograms of local angular model deviation estimated on the basis of equation (1); that is, all BRF values are accumulated over all illumination geometries and at two wavelengths. The top left, top right, bottom left, and bottom right panels display results obtained for the total BRF, the multiplescattering, the single uncollided, and the single collided scattering contributions, respectively. Results correspond to simulations performed with respect to the floating sphere scene that was part of RAMI Phase 1.

infrared wavelength only. It thus suggests quite logically that the performance of the RGM model can be improved by upgrading the estimates of the single-scattering contribution. It will be noted that the results obtained during this second phase of RAMI show very significant improvements with respect to those displayed after the completion of Phase 1 in 1999. These are primarily due to efforts made by the RAMI participants to improve their models in terms of their ability to represent accurately the radiation transfer regimes in plant canopies, as well as their care in implementing properly the relevant computing schemes.

\subsection{Three-Dimensional Structurally Heterogeneous Scenes}

\subsubsection{RAMI Phase 1 Three-Dimensional}

\section{Heterogeneous Scene}

[34] The first set of 3-D experiments in Phase 2 is identical to that already proposed under Phase 1 (see section 3.2 of Pinty et al. [2001]). The results presented here thus corre- spond either to computations with updated models that had already participated in RAMI Phase 1 , or to new and additional simulations not performed previously. In this case, the participating groups obviously had advance knowledge of the results generated earlier. A total of nine models participated in the simulation of the BRF fields of a structurally heterogeneous scene made up of leaves clumped into "floating" spheres (see Figure 1b and Table 5 of Pinty et al. [2001]). This scene constitutes a first level of complexity when structurally heterogeneous cases are considered.

[35] Figure 7 displays the results generated by the nine models in the principal and cross planes, at the red and nearinfrared wavelengths and for two illumination zenith angles of $20^{\circ}$ and $50^{\circ}$, respectively. Taken together, the models appear to agree much more on the overall shape of the BRF fields than on their amplitudes. Large variations, by as much as a factor of 2, can be seen between the simulation results. Many reasons could explain these variations. For instance, in the case of geometric-optical models, it may be difficult 
to account for a large number of nonoverlapping objects. The geometric-optical models are currently being improved in order to account for the nonoverlapping component, especially under low-Sun-zenith-angle conditions, by incorporating a nonoverlapping factor and/or using different tree crown distribution functions and, to separate the single- and multiple-scattering contributions. The simplicity of the geometric-optical models makes them, however, attractive candidates for inversion purposes. The experimental conditions are anyway such that the discrepancies at the nearinfrared wavelength (right panels) are mostly due to the different techniques and thus assumptions implemented in solving the multiply scattered and the single collided components of the radiation transfer regime. By contrast, the differences in the simulation of the single collided and uncollided contributions explain most of the variabilities shown at the red wavelength (left panels). Nevertheless, it remained difficult to fully assess the behavior of some of the participating models because results were provided only for a subset of the entire set of proposed experiments.

[36] Figure 8 shows four histograms of local angular model deviations, the $\delta_{m}\left(\theta_{v}\right)$ values estimated from equation (1), assessed for the entire set of experiments, corresponding to the simulation of a total of $608 \mathrm{BRF}$ values, proposed in the case of the scene composed of "floating" spheres. The histograms refer to the total BRF fields and the three separate radiative transfer components. Some models were left out at this stage since they did not perform a large enough subset of the suggested experiments to be part of this detailed analysis. The bimodal nature of the histogram in the case of the multiple-scattering contribution, as well as the presence of long tails in the single collided and uncollided contributions, indicate the presence of one or more "outliers"; that is, models producing BRF values that are distinctly different from those produced by the other RAMI models. A detailed analysis reveals that three RAMI models are producing these histogram features. Figure 9 exhibits the same histogram in total BRFs, equivalent to the upper left panel of Figure 8, after removing the contribution from these "outliers." It is very encouraging to note that (1) the five remaining models together deviate only by about 1 to $3 \%$ of their BRF values and (2) this averaged deviation has been reduced since the first phase of RAMI that took place in 1999.

\subsubsection{Coniferous Forest Scene}

[37] This set of experiments permitted to cover an interesting range of geophysical situations from the perspective of radiation transfer simulation. Indeed, this 3-D scene challenged the models by providing two kinds of structural organization, one imposed by the topography and the other induced by the specific shapes of the trees, as well as a strong spectral contrast between the foliage and the background, in two distinct spectral bands (red and nearinfrared).

[38] These experiments constitute a very demanding test of the ability of the models to effectively describe radiation transfer in complex environments. Although the uncollided contribution (radiation scattered once by the soil in the direction of the measurement and not interacting with the foliage) is prominent at the red wavelength, the contributions from neither the single collided (by the trees only) nor the multiply scattered (by the tree-soil system) components

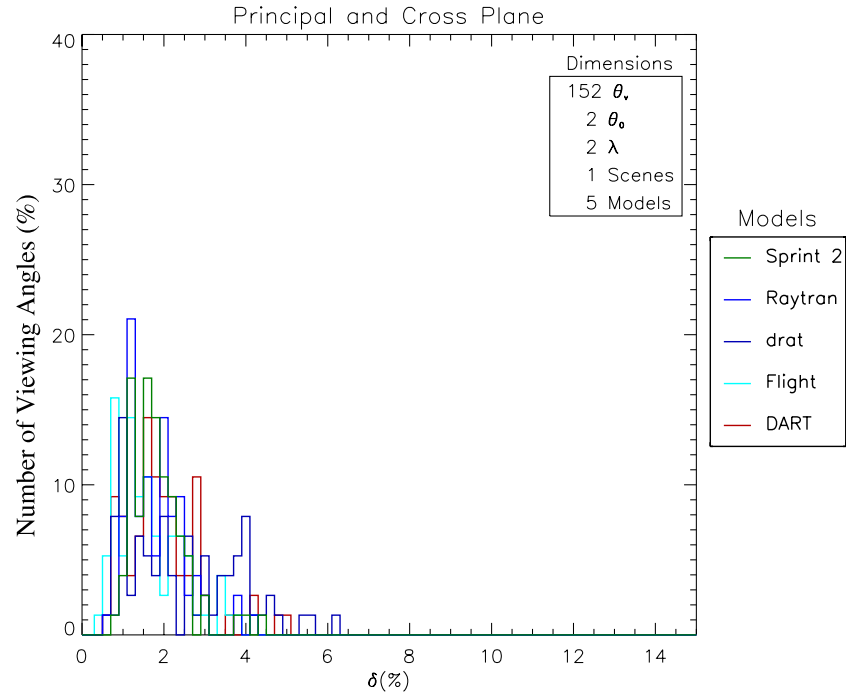

Figure 9. Histogram of local angular model deviation with respect to the total BRF values estimated from five models on the basis of equation (1); that is, all BRF values are accumulated over all illumination and viewing geometries, at two wavelengths. Results correspond to simulations performed with respect to the floating sphere scene that was part of RAMI Phase 1.

are negligible. In the near-infrared, the multiple-scattering contribution is of the same order of magnitude as the uncollided term while the single collided term is about one tenth of the other two. These sets of spectral and structural properties yield typical bell-shaped BRFs [Pinty et al., 2002] at both wavelengths, as can be observed currently by the Multiangle Imaging Spectroradiometer (MISR) instrument over boreal forests [see, e.g., Gobron and Lajas, 2002].

[39] Figure 10 exhibits the total BRF results generated by some of the RAMI Phase 2 models in the principal and cross planes, at the two suggested wavelengths (see Table 3). Although these four models (other 3-D RT models did not participate in these experiments) produced quite analogous bell-shaped BRF at both wavelengths, the discrepancies between the simulation results can be as large as 0.1 for a relatively wide range of exiting angles. A detailed inspection of results obtained while performing other experiments for that scene, reveals that (1) the DART model tends to overestimate the multiple-scattering as well as the single uncollided components, especially in the exact backscattering direction, (2) the Drat model overestimates the single uncollided component in the red wavelength, and (3) the Sprint-2 and Raytran models are essentially indistinguishable for the entire set of experiments proposed for this synthetic coniferous forest scene.

[40] The discrepancies observed in Figure 10 between the RT models may have various origins, including remaining software inaccuracies and/or fundamental model limitations with regards to the representation of scene architecture. It was, for instance, technically difficult for the participating version of the DART model to process such a large scene with the locally available computer resources without approximating the description of the canopy structure. An 
Principal plane
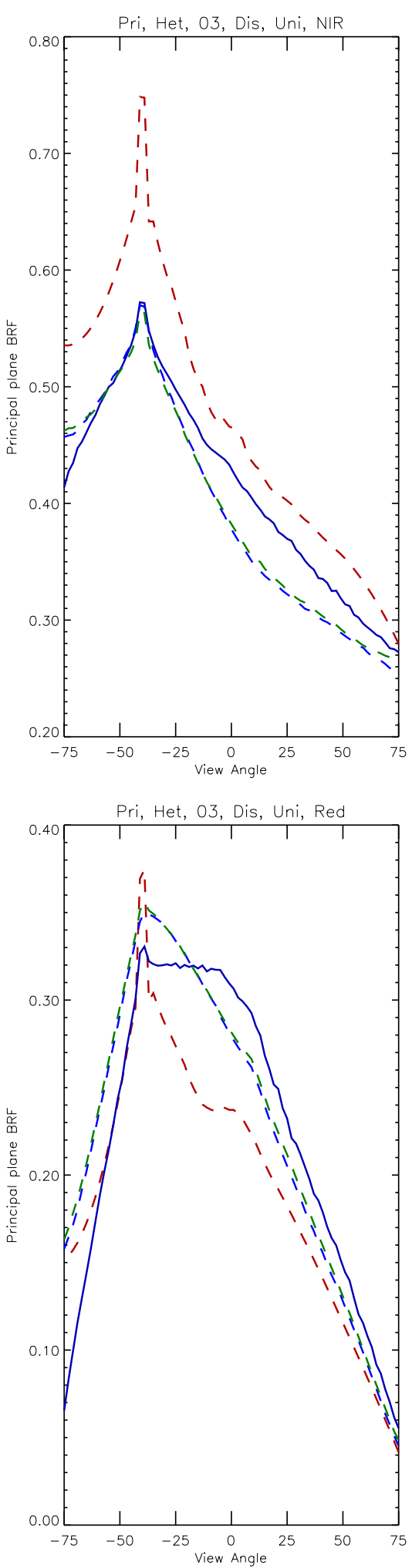

Cross plane

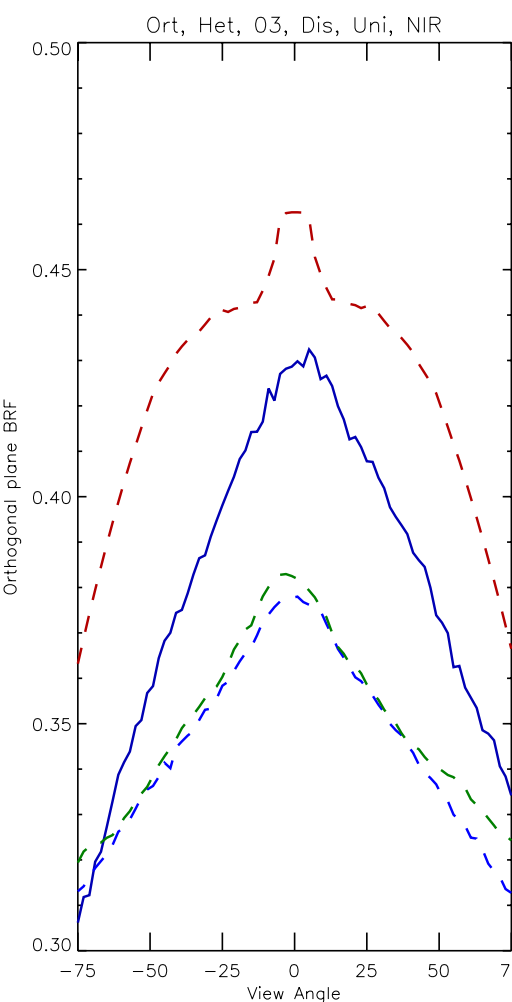

- Sprint 2
- Raytran

- drat

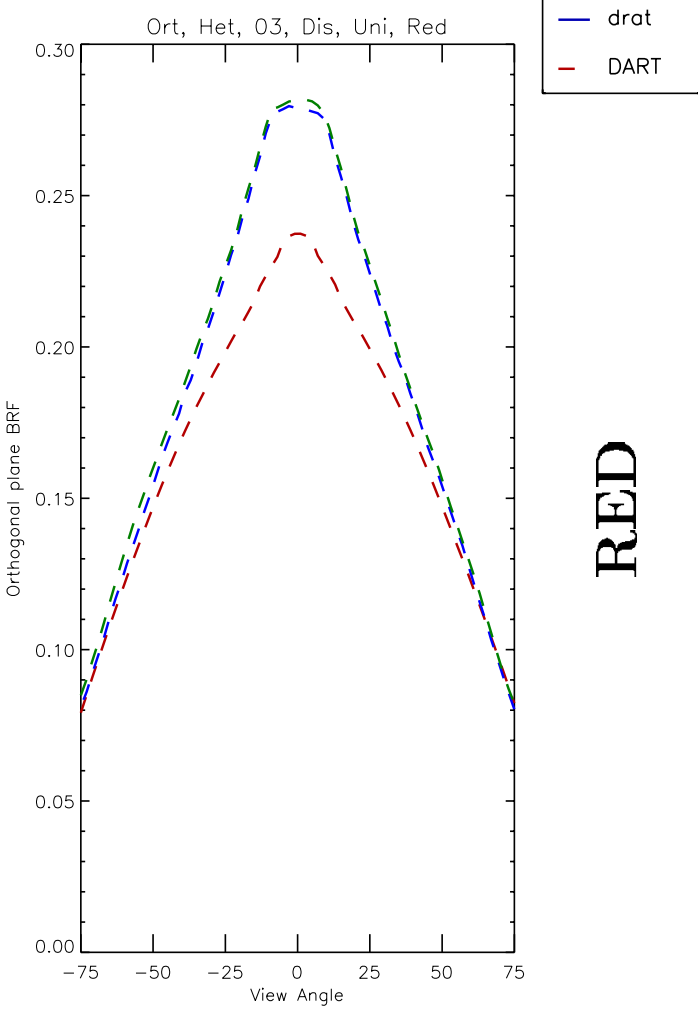

Figure 10. Total BRF results from the RAMI Phase 2 models plotted for the near-infrared (top panels) and red (bottom panels) wavelengths. The two left (right) panels correspond to results obtained in simulating the radiation transfer regime in the principal (cross) planes. The conditions of the experiments are given on top of each panel using the following convention: Pri (Ort) identifies the principal (cross) plane, Het is for heterogeneous scene, Dis stands for discrete scatterers, and Uni corresponds to uniform leaf angle distribution. Results correspond to simulations performed with respect to the coniferous tree scene. 
updated version of the DART model incorporating the use of dynamic instead static memory-arrays is currently under testing and will participate in future RAMI activities. For its part, the Drat model provides an estimate of the BRF at a point located within a finite distance from the scene, with a detector whose dimension varies with the observation angle. This implies that, in the presence of topography, the Drat model may have sampled the BRF field somewhat differently from other models. The radiation transfer regime per se could thus be correctly simulated by the model but the sampling procedure implemented in the model may not have strictly conformed to the RAMI protocol. For instance, Drat simulations are performed with an orthographic projection, so that rays exiting the scene can be considered parallel. If the scene is repeated infinitely in all directions (in Drat simulations the scene is replicated many times but not infinitely), the total radiation arriving at the image plane should be identical to that measured by a sensor placed at infinity, sampling the whole viewing hemisphere. This is purely an implementation difference between forward and reverse Monte-Carlo ray tracing models. While this difference should not (theoretically) result in different BRF values, more detailed testing may be required to identify the precise cause of observed variations in modeled BRFs. Incidentally, it is worth noting that the net effect of this particular topography on the BRF values exiting the scene at both the near-infrared and red wavelengths was found to be of limited importance, as illustrated in Figure 11. It can readily be seen that the changes in the BRF fields simulated with and without topography are small enough to be within the entire range of variability of the BRF results produced by the four participating models. This conclusion holds true for the entire set of experiments proposed for that particular scene.

\subsubsection{Structurally and Spectrally Mixed Vegetation Scene}

[41] The simulation of the radiation transfer regime in this second heterogeneous scene constitutes a more classical case study than the previous one. Indeed, the complexity of the scene was limited to the overall canopy architecture. The scattering elements (the "leaves" and the underlying soil) were represented through their traditional geometrical and spectral properties. The difficulty and interest of this experiment arose from the requirement to perform simulations for three successive spatial resolutions $\left(270 \times 270 \mathrm{~m}^{2}, 90 \times\right.$ $90 \mathrm{~m}^{2}$ and $30 \times 30 \mathrm{~m}^{2}$ ), as mentioned earlier. This set of experiments permitted to evaluate the robustness of the participating models in an entirely new domain, made relevant by the existing or planned availability of spaceborne instruments operating at these or similar spatial resolutions.

[42] Figure 12 exhibits the BRF results generated by five RT models in the near-infrared, in the principal and cross planes (left and right panels), and at the three given spatial resolutions (top, middle and bottom panels), respectively. These models exhibit general agreement at the 270 and $90 \mathrm{~m}$ resolutions, except for near-nadir observation angles, where a small bell-shaped reflectance pattern localized near the nadir overcomes the overall bowl-shape pattern. At the highest resolution $(30 \mathrm{~m})$, the models differ somewhat more between each other.

[43] The FRT model does not permit an explicit representation of spatial resolution; its results are thus reported

\section{Principal plane at the near-infrared wavelength}

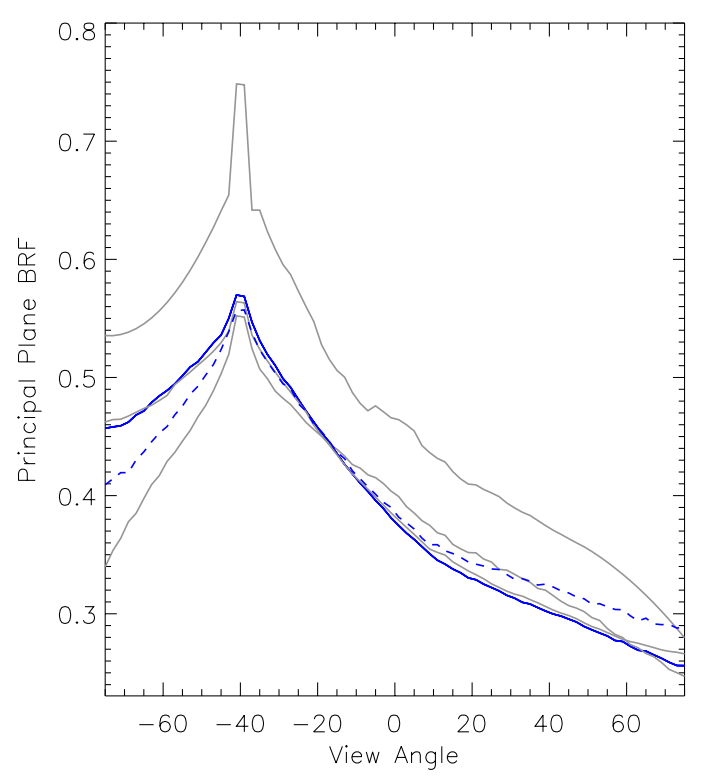

Figure 11. Total BRF results generated in the principal plane at the near-infrared wavelength by the Raytran model with (full blue line) and without (dashed blue line) incorporating topography in the coniferous tree scene. BRF results obtained from other RAMI models (shown in the top left panel of Figure 10) are also reproduced (grey lines).

for the $270 \mathrm{~m}$ resolution only, where the model is presumably more appropriate, given the design assumptions. The observed deviations between the FRT model and the others at large viewing angles are believed to result from the use of a two-stream approximation, suitable for horizontally homogeneous canopies, to simulate the multiple-scattering component. This assumption yields a slightly smoother multiply scattered radiance field than those derived by models implementing a scheme that accounts explicitly for spatial heterogeneity. The agreement (not shown) between the FRT model and the other participating models is, however, quite good for the single collided and uncollided components of the total exiting radiance fields.

[44] The slight but consistent overestimate of the BRF in the backward direction and in the principal plane by DART is posited to result from the contribution of its simulated single collided component.

[45] The Sprint-2 model generates small but unexpected variations in the close to nadir BRF field estimated in the cross plane at the intermediate spatial resolution. This model also generates almost systematically the lowest BRF values at the highest spatial resolution, for subtle reasons that remain to be investigated.

[46] In general, the discrepancies between the models are largely attributed to the single collided and uncollided components. At the highest spatial resolution considered here, however, differences between model results are also due to the multiple-scattering contribution. In fact, as the spatial resolution increases, the scene statistics are no longer conserved and the precise location of the individual scat- 


\section{Principal plane}
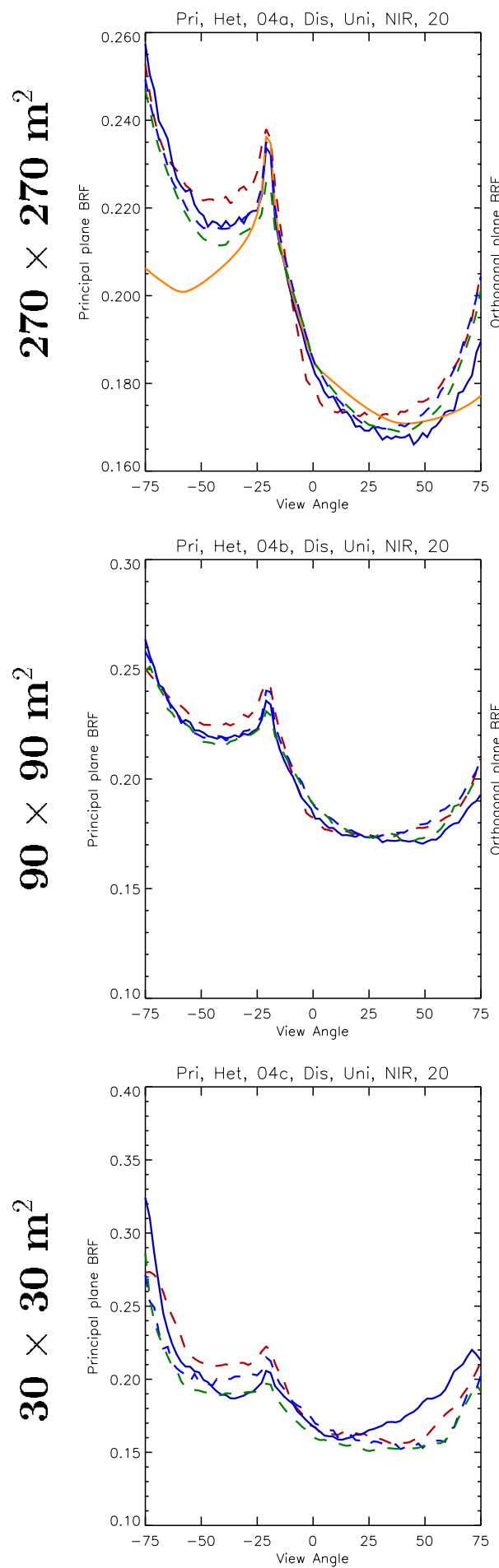

Cross plane
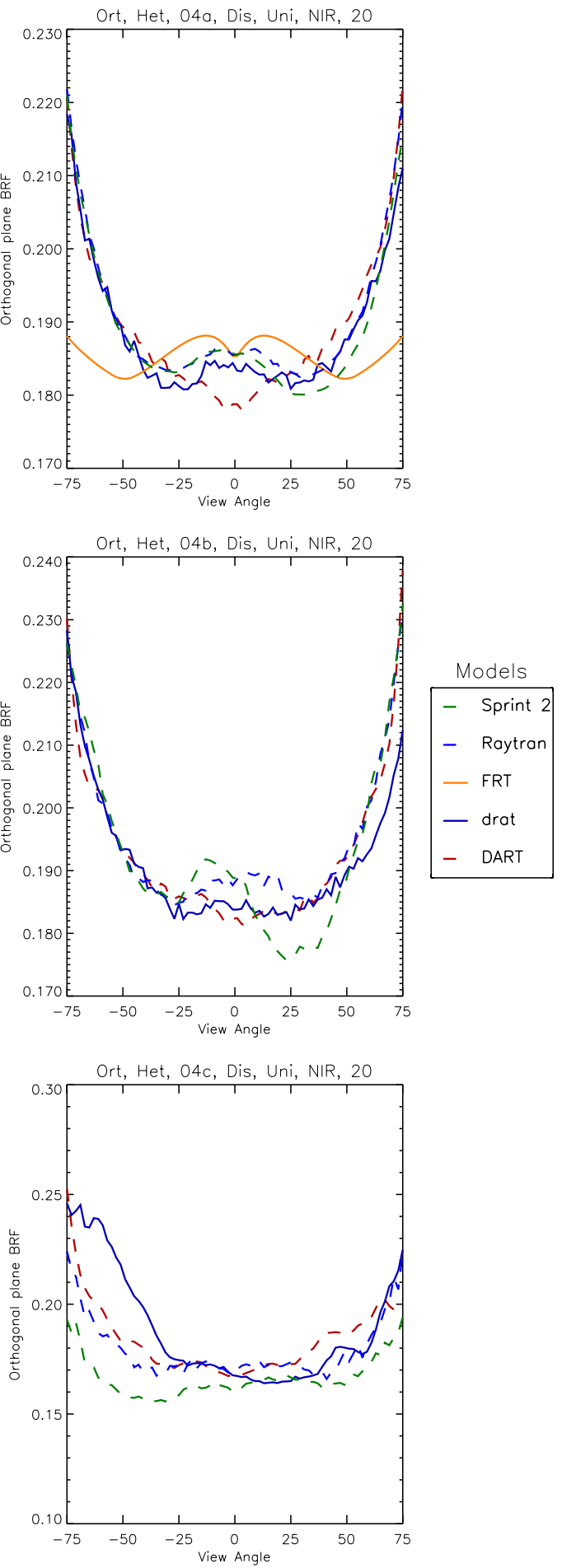

Figure 12. Total BRF values simulated in the principal (cross) plane on the left (right) panels at spatial resolutions of $270 \times 270 \mathrm{~m}^{2}$ (top panels), $90 \times 90 \mathrm{~m}^{2}$ (middle panels), and $30 \times 30 \mathrm{~m}^{2}$ (bottom panels). The conditions of the experiments are given on top of each panel using the same convention as before. Results correspond to simulations performed with respect to the structurally and spectrally mixed vegetation scene.

terers composing the sampled scene control the overall multiply scattered radiance fields.

[47] This set of experiments illustrates quantitatively the difficulties associated with the solution of radiative transfer problems in plant canopies at high spatial resolution. Indeed, and as should be expected, the higher the spatial resolution, the higher the sensitivity of the results to the spatial distribution and properties of the individual scattering elements.

[48] The BRF signatures of heterogeneous canopies are thus dependent on spatial resolution itself, to the extent that 


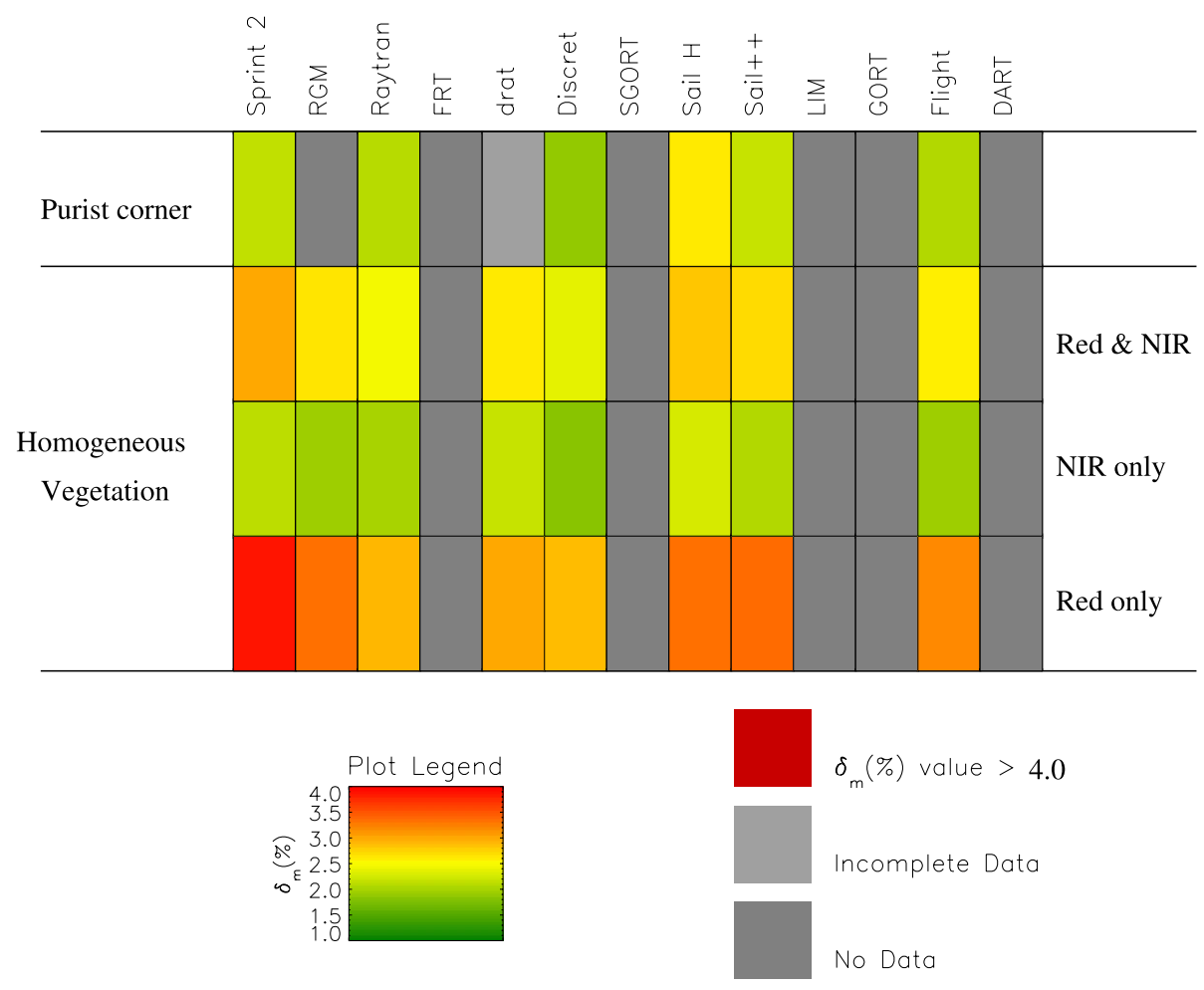

Figure 13. Table indicating both the level of participation of the RAMI models in the proposed sets of experiments and their overall performance. The dark (light) grey color highlights the experiments that were not (partially) performed. The other three colors express, for each model and experiment, the value of the global model deviation that results from a calculation of the mean value of the local angular deviations estimated on the basis of equation (1). This table summarizes the results obtained for the structurally homogeneous scenes.

the statistics of the canopy attributes are not conserved with changes in spatial resolution [e.g., Gerard and North, 1997; Widlowski, 2002]. Accordingly, the range of BRF shapes and amplitudes that can be associated with a typical biome type can be rather large, depending on the spatial resolution of the instrument. This further implies that the reliability of a particular solution to the inverse problem of characterizing the environment on the basis of remote sensing observations decreases with the increasing spatial resolution of the instrument.

\subsection{Summary of Model Participation and Performances}

[49] One of the primary objectives of RAMI is to quantify the variability of the BRFs simulated by a particular RT model with respect to the values generated by all other participating models, within the context of a specific set of tests. For this to work effectively, it is essential for each model to conduct the complete set of computations suggested within each test category (e.g., structurally homogeneous and heterogeneous scenes). Figures 13 and 14 summarize the actual rate of participation in each series of tests during the second phase of RAMI, as well as the global performance of the modeling community for the structurally homogeneous and heterogeneous scenes, respectively. The color coding highlights both model participation and model performance within each series of experiments. The dark (light) grey color highlights the experiments that were not (or only partially) performed.
The other three colors express, for each model and experiment, the value of the global model deviation that results from a calculation of the mean value of the local angular deviations estimated on the basis of equation (1) (see equation (3) of Pinty et al. [2001]). It is important to reiterate that the local and global deviation metrics represent only the deviations of each model with respect to all other models taken together. This metrics thus represents the current performance of the modeling community as a group. The extent of the "grey" areas in these figures indicates that many models did not perform all proposed experiments, thereby preventing an effective assessment in that particular context. Future efforts should thus aim toward more exhaustive testing, by ensuring that all participating models conduct a more complete battery of simulations, and toward improving the models so that the color coding turns green (agreement on the probable BRF field values) rather than red (disagreement).

\section{Concluding Remarks and Future Perspectives for RAMI}

[50] The Radiation Transfer Model Intercomparison (RAMI) international benchmarking exercise has reached a new level of maturity and now proceeds on its own momentum. Indeed, the number of participants and RT models evaluated within the RAMI context has significantly grown over time. The main findings after the second phase of this activity can be summarized as follows: 


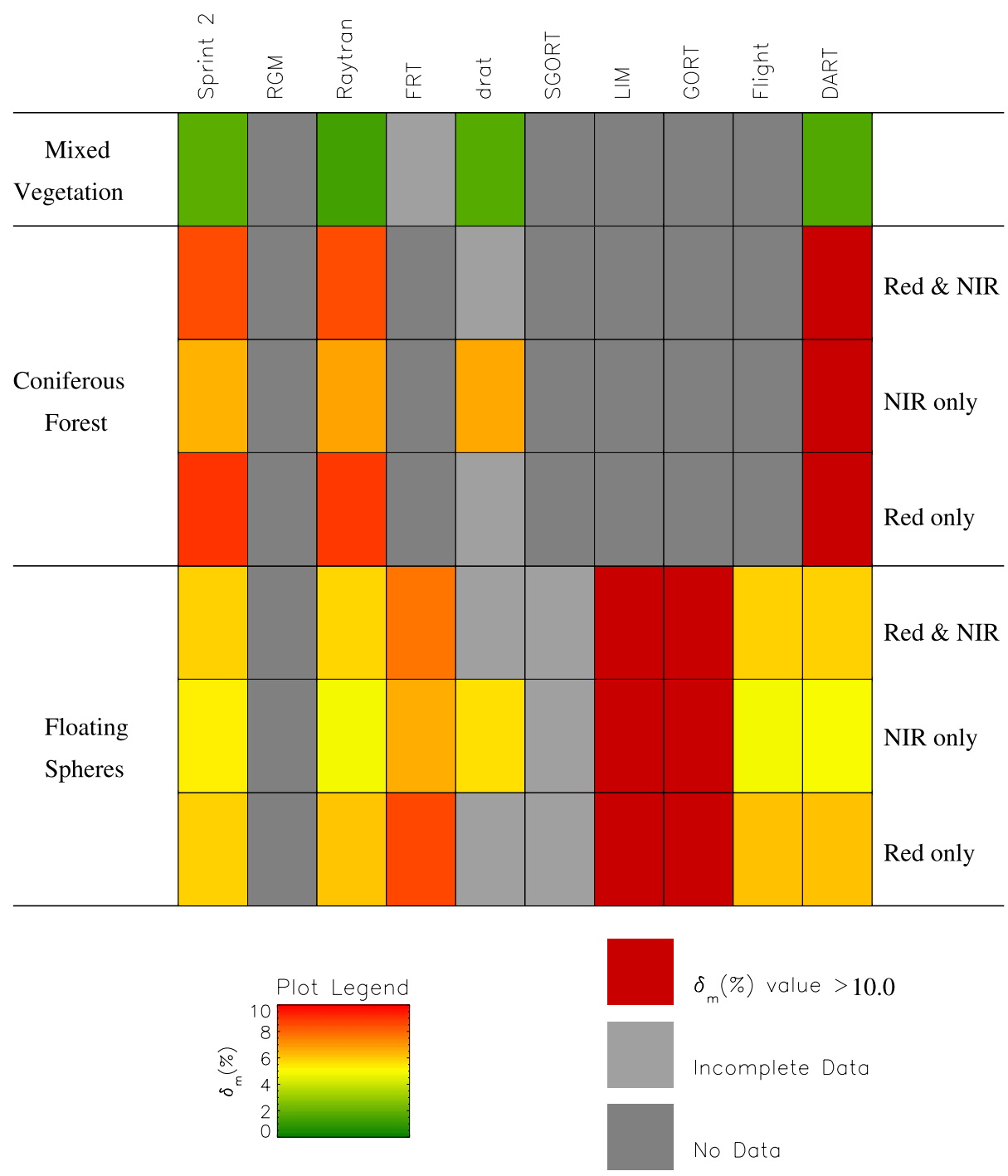

Figure 14. Same as Figure 13 except for the case of structurally heterogeneous scenes.

[51] 1. The series of experiments designed during Phase 1 of RAMI to test models over structurally homogeneous scenes has become de facto a baseline to evaluate both simple 1-D and more elaborate 3-D RT models. The reconduction of tests carried out previously can serve both to bring existing or new models on board, and to document the progress made in time. In fact, the improved agreement between some of the participating models (compared to Phase 1) in the case of simple geophysical scenes, which occurred despite a wide diversity in approach, methodology and implementation, shows that improvements are still possible, or that errors have been corrected, or both. This is a key motivation and justification for the maintenance of RAMI as a continuing, if evolving, activity.

[52] 2. The definition of new and challenging experiments plays an important role in that these simulations are necessarily performed "blindly" by the participants, who do not know what to expect a priori from such exercises. This provides a unique opportunity to test all participating models in ways that are most likely to unearth unexpected differences.

[53] 3. Some of the 3-D models are now indistinguishable from a radiation transfer point of view over particular sets of experiments, in the sense that the differences between their outputs are less than a few percent of the BRF values themselves. Although this does not define the "true solution," the collective behavior of these models certainly constitutes a reasonable "most credible solution." Such models can therefore serve as references for the benchmarking of other models. In the future, the RAMI community may decide to deliver "RAMI certificates of compliance" to models that have been shown to pass tests (such as satisfying the conservation of energy for conservative scattering conditions within specified bounds) and to substantially agree with the prevailing consensus. It is thus appropriate for all 1-D and 3-D RT models participating in the RAMI exercise to perform the ensemble of tests proposed in the case of structurally homogeneous scenes whenever it is technically feasible.

[54] 4. The latest incarnation of RAMI has permitted to investigate the performance of advanced 3-D RT models in describing the reflectance of both simple homogeneous and complex heterogeneous scenes, and to extend the pertinence of this benchmarking exercise to hitherto uncharted areas.

[55] 5. The remaining and sometimes substantial differences and discrepancies between these 3-D models over 
complex heterogeneous scenes have effectively placed a limit on their current reliability, as well as defined a concrete goal for the near future. Specifically, the divergence in their ability to properly describe the BRF field at various spatial resolutions constitutes a serious challenge and an invitation to investigate emerging issues.

[56] 6. As models evolve and tend to agree with each other it will become important to design ever more advanced experiments to investigate the remaining differences. In particular, the issue of whether these differences arise from errors in codes or subtle differences in software implementation or combination of both are not yet fully explored. For example, scene representation tolerance limits, e.g., the specification of the exact locations of all scatterers used within the 3-D models may cause slight differences depending on the scene being rendered. The need to move to more detailed intercomparison tests is certainly positive as the RAMI exercise has seen model agreement improve significantly between the first and the second phase.

[57] 7. The methodology proposed earlier (in Phase 1) to evaluate the performance of any given model with respect to the collective achievements of the group of participating models has been well received and is emerging as a new standard in its own right.

[58] 8. Last but not least, RAMI remains a voluntary, community-driven activity open to all participants willing to perform the proposed computations. All models have benefited from this exercise in one way or another, and all investigators who have genuinely contributed to it have been systematically associated with the analysis and publication of the results. RAMI has permitted to create productive links within the global RT community, and has allowed scientists to collaborate across continents.

[59] RAMI will likely evolve on various fronts. If the primary beneficiaries of this exercise are the participants who get an opportunity to benchmark and therefore to improve their models, the results should be of interest to the scientific community at large and to current or prospective users of these models in particular. The inclusion of more and more complex scenes will stimulate the RT modeling community to keep improving the tools of the trade and to provide reliable and accurate models useful for the practical solution of problems in a wide range of applications. Some selection of the proposed tests may become the basis upon which to base the issuance of certificates. RAMI may well become a more dynamic process, where participation is not restricted to specific periods of intense activity, but, rather, an on-going and evolving method of evaluation and confrontation for participating models. Analyses of accumulated results and syntheses of the state of the art will continue to be unveiled every 2 to 3 years, perhaps on the occasion of future editions of the International Workshop on Multiangular Measurements and Models, and to be published in the refereed literature.

[60] Acknowledgments. Phase 2 of RAMI took place as part of the preparations for the IWMMM-3 conference organized in Steamboat Springs, Colorado, by Anne W. Nolin of the National Snow and Ice Data Center in Boulder, Colorado, USA, and David J. Diner of the Jet Propulsion Laboratory, California Institute of Technology, Pasadena, California, USA. The conference was jointly sponsored by (1) the National Aeronautics and
Space Administration (NASA), (2) the U.S. Department of Energy, and (3) the Colorado/Cooperative Institute for Research in Environmental Sciences (CIRES). The definition of the RAMI experiments, the coordination of the participants, the analysis of the simulations, and the publication of the results would not have been possible without the support of the Global Vegetation Monitoring unit of the Institute for Environment and Sustainability at the Joint Research Centre, an institution of the European Commission. The stimulating exchanges of ideas with other scientific communities, in particular with I3RC, are also gratefully acknowledged. The critical comments and constructive suggestions of Anthony Davis on a preliminary draft of this manuscript are deeply appreciated.

\section{References}

Gastellu-Etchegorry, J.-P., V. Demarez, V. Pinel, and F. Zagolski (1996), Modeling radiative transfer in heterogeneous 3-D vegetation canopies, Remote Sens. Environ., 58, 131-156.

Gerard, F. F., and P. R. J. North (1997), Analyzing the effect of structural variability and canopy gaps on forest BRDF using a geometric-optical model, Remote Sens. Environ., 62, 46-62.

Gobron, N., and D. Lajas (2002), A new inversion scheme for the RPV model, Can. J. Remote Sens., 28, 156-167.

Gobron, N., B. Pinty, M. M. Verstraete, and Y. Govaerts (1997), A semidiscrete model for the scattering of light by vegetation, J. Geophys. Res., $102,9431-9446$

Govaerts, Y., and M. M. Verstraete (1998), Raytran: A Monte Carlo ray tracing model to compute light scattering in three-dimensional heterogeneous media, IEEE Trans. Geosci. Remote Sens., 36, 493-505.

Irvine, W. M. (1966), The shadowing effect in diffuse reflection, J. Geophys. Res., 71, 2931-2937.

Knyazikhin, Y. V., and A. L. Marshak (1991), Fundamental equations of radiative transfer in leaf canopies, and iterative methods for their solution, in Photon-Vegetation Interactions, edited by R. Myneni and J. Ross, pp. 9-43, Springer-Verlag, New York.

Knyazikhin, Y. V., A. L. Marshak, and R. B. Myneni (1992), Interaction of photons in a canopy of finite-dimensional leaves, Remote Sens. Environ., 39, 61-74.

Knyazikhin, Y. V., A. L. Marshak, and R. B. Myneni (2004), Three-dimensional radiative transfer in vegetation canopies, in Three-Dimensional Radiative Transfer in the Cloudy Atmosphere, edited by A. Davis and A. Marshak, Springer-Verlag, New York, in press.

Kuusk, A., and T. Nilson (2000), A directional multispectral forest reflectance model, Remote Sens. Environ., 72, 244-252.

Lewis, P. (1999), Three-dimensional plant modelling for remote sensing simulation studies using the botanical plant modelling system, Agron. Agric. Environ., 19, 185-210.

Li, X., A. H. Strahler, and C. E. Woodcock (1995), A hybrid geometric optical radiative transfer approach for modeling albedo and directional reflectance of discontinuous canopies, IEEE Trans. Geosci. Remote Sens., 33, 466-480.

Ni, W., X. Li, C. E. Woodcock, M. R. Caetano, and A. Strahler (1999), An analytical hybrid GORT bidirectional reflectance model for discontinuous plant canopies, IEEE Trans. Geosci. Remote Sens., 37, 987-999.

Nilson, T., and A. Kuusk (1989), A reflectance model for the homogeneous plant canopy and its inversion, Remote Sens. Environ., 27, 157-167.

North, P. R. J. (1996), Three-dimensional forest light interaction model using a Monte Carlo method, IEEE Trans. Geosci. Remote Sens., 34, $946-956$

Pinty, B., and M. M. Verstraete (1998), Modeling the scattering of light by vegetation in optical remote sensing, J. Atmos. Sci., 55, 137-150.

Pinty, B., et al. (2001), The Radiation Transfer Model Intercomparison (RAMI) exercise, J. Geophys. Res., 106, 11,937-11,956.

Pinty, B., J.-L. Widlowski, N. Gobron, M. M. Verstraete, and D. J. Diner (2002), Uniqueness of multi-angular measurements. Part 1: A subpixel surface heterogeneity indicator from MISR, IEEE Trans. Geosci. Remote Sens., 40, 1560-1573.

Qin, W., and S. A. W. Gerstl (2000), 3-D scene modeling of semi-desert vegetation cover and its radiation regime, Remote Sens. Environ., 74, $145-162$

Ross, J. (1981), The Radiation Regime and Architecture of Plant Stands, 391 pp., Dr. W. Junk, Norwell, Mass.

Saich, P., P. Lewis, M. Disney, and G. Thackrah (2001), Comparison of Hymap/E-SAR data with models for optical reflectance and microwave scattering from vegetation canopies, in Proceedings of Third International Workshop on Retrieval of Bio- and Geo-Physical Parameters From SAR data for Land Applications, p. 427, Univ. of Sheffield, Sheffield, UK.

Shultis, J. K., and R. B. Myneni (1988), Radiative transfer in vegetation canopies with anisotropic scattering, J. Quant. Spectrosc. Radiat. Transfer, 39, 115-129. 
Thompson, R. L., and N. S. Goel (1998), Two models for rapidly calculating bidirectional reflectance: Photon spread (ps) model and statistical photon spread (sps) model, Remote Sens. Rev., 16 , $157-207$.

Verhoef, W. (1984), Light scattering by leaf layers with application to canopy reflectance modeling: The SAIL model, Remote Sens. Environ., $16,125-141$

Verhoef, W. (1985), Earth observation modeling based on layer scattering matrices, Remote Sens. Environ., 17, 165-178.

Verhoef, W. (1998), Theory of radiative transfer models applied to optical remote sensing of vegetation canopies, Ph.D. thesis, Wageningen Univ., Wageningen, Netherlands.

Verhoef, W. (2002), Improved modelling of multiple scattering in leaf canopies: The model SAIL++, in Proceedings of the First Symposium on Recent Advances in Quantitative Remote Sensing, Torrent, Spain, edited by A. Sobrino, pp. 11-20, Univ. of Valencia, Valencia, Spain.

Verstraete, M. M. (1987), Radiation transfer in plant canopies: Transmission of direct solar radiation and the role of leaf orientation, J. Geophys. Res., 92, 10,985-10,995.

Verstraete, M. M., B. Pinty, and R. E. Dickinson (1990), A physical model of the bidirectional reflectance of vegetation canopies: 1. Theory, J. Geophys. Res., 95, 11,765-11,775.

Widlowski, J.-L. (2002), Extracting quantitative sub-pixel heterogeneity information from optical remote sensing data, Rep. EUR 20236 EN, Joint Res. Cent., Ispra, Italy.

M. Disney and P. Lewis, Center for Terrestrial Carbon Dynamics (CTCD), Department of Geography, University College London, 26
Bedford Way, London WC1H 0AP, UK. (mdisney@geography.ucl.ac.uk; plewis@geog.ucl.ac.uk)

F. Gascon, Land Surface Unit, European Space Agency, Keplerlaan 1, NL-2200AG, Noordwijk, Netherlands. (ferran.gascon@esa.int)

J.-P. Gastellu, Centre d'Études Spatiales de la Biosphère, 18 Avenue Edouard Belin, F-31055 Toulouse, France. (jean-philippe.gastellu@cesbio. cnes.fr)

N. Gobron, B. Pinty, M. Taberner, M. M. Verstraete, and J.-L. Widlowski, Global Vegetation Monitoring Unit, IES, EC Joint Research Centre, TP 440, via E. Fermi, I-21020 Ispra (VA), Italy. (nadine.gobron@jrc.it; bernard.pinty@jrc.it; malcolm.taberner@jrc.it; michel.verstraete@jrc.it; jean-luc.widlowski@jrc.it)

L. Jiang, X. Li, L. Su, S. Tang, H. Wang, J. Wang, G. Yan, and H. Zang, Department of Geography, Beijing Normal University, Beijing 100875, China. (su lihong@hotmail.com; gjyan@bnu.edu.cn)

A. Kuusk and T. Nilson, Tartu Observatory, EE-61602 Tõravere, Tartumaa, Estonia. (andres@aai.es; nilson@aai.ee)

W. Ni-Meister, Department of Geography, Hunter College, City University of New York, New York, NY 10021, USA. (wenge@geo. hunter.cuny.edu)

P. North, Climate and Land Surface Systems Interaction Centre (CLASSIC), Department of Geography, University of Wales, Singleton Park, Swansea SA2 8PP, UK. (p.r.j.north@swansea.ac.uk)

W. Qin, NASA Goddard Space Flight Center, Code 916, Greenbelt, MD 20771, USA. (wenhan_qin@ssaihq.com)

R. Thompson, Alachua Research Institute, 8202 NW 156 Avenue, P. O. Box 1920, Alachua, FL 32616, USA. (richard.thompson@prodigy.net)

W. Verhoef, Remote Sensing Department, National Aerospace Laboratory NLR, P. O. Box 153, 8300 AD Emmeloord, Netherlands. (verhoef@ nlr.nl) 